\title{
High-energy effective action from scattering of QCD shock waves
}

\author{
Ian Balitsky \\ JLab \& ODU \\ Minneapolis, 13 May 06
}




\section{High-energy scattering as a collision of shock waves}

A typical hadron-hadron collision viewed from the c.m. frame has the form of scattering of two shock waves.

Regge limit: $E \gg$ everything else
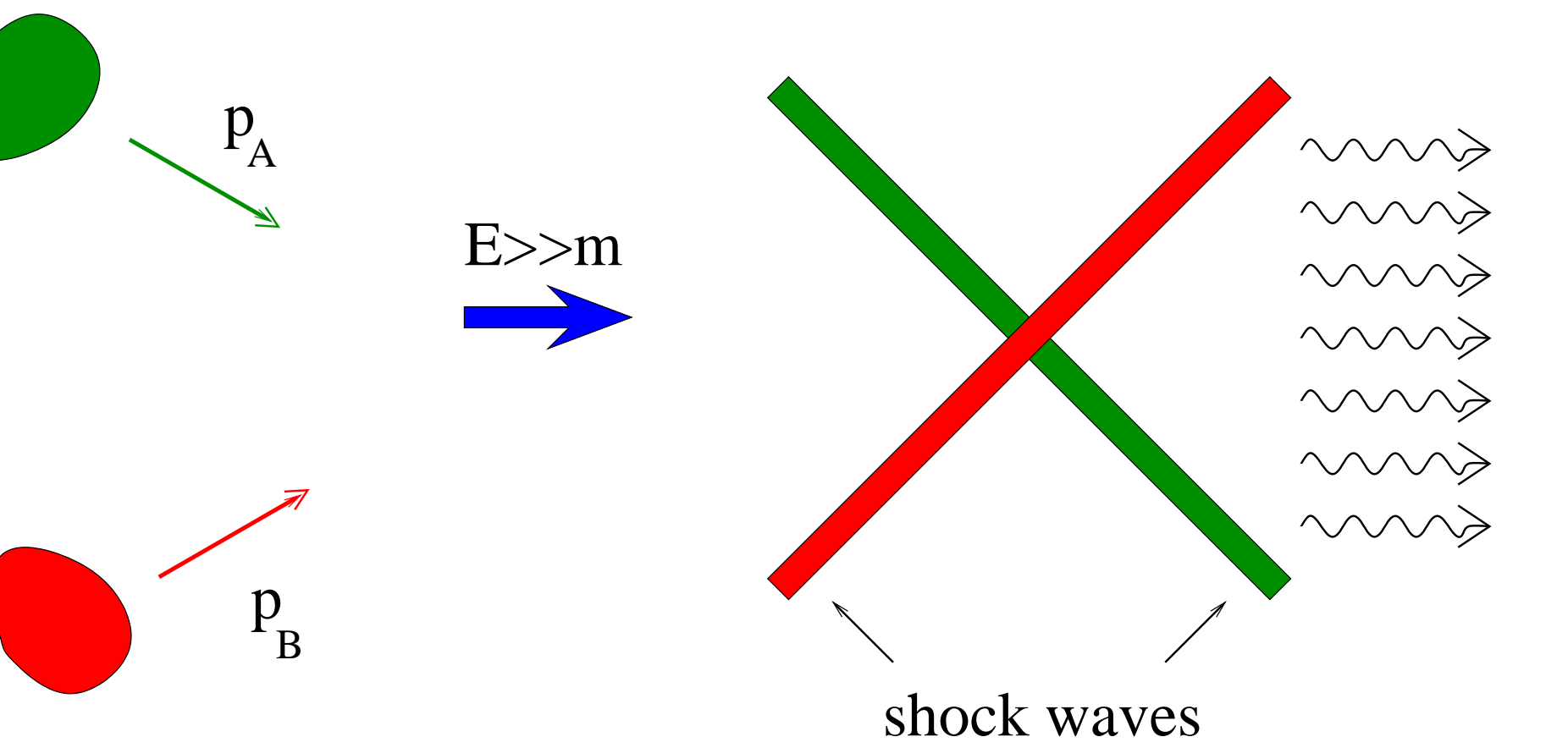


\section{High-energy scattering as a collision of shock waves}

A typical hadron-hadron collision viewed from the c.m. frame has the form of scattering of two shock waves.
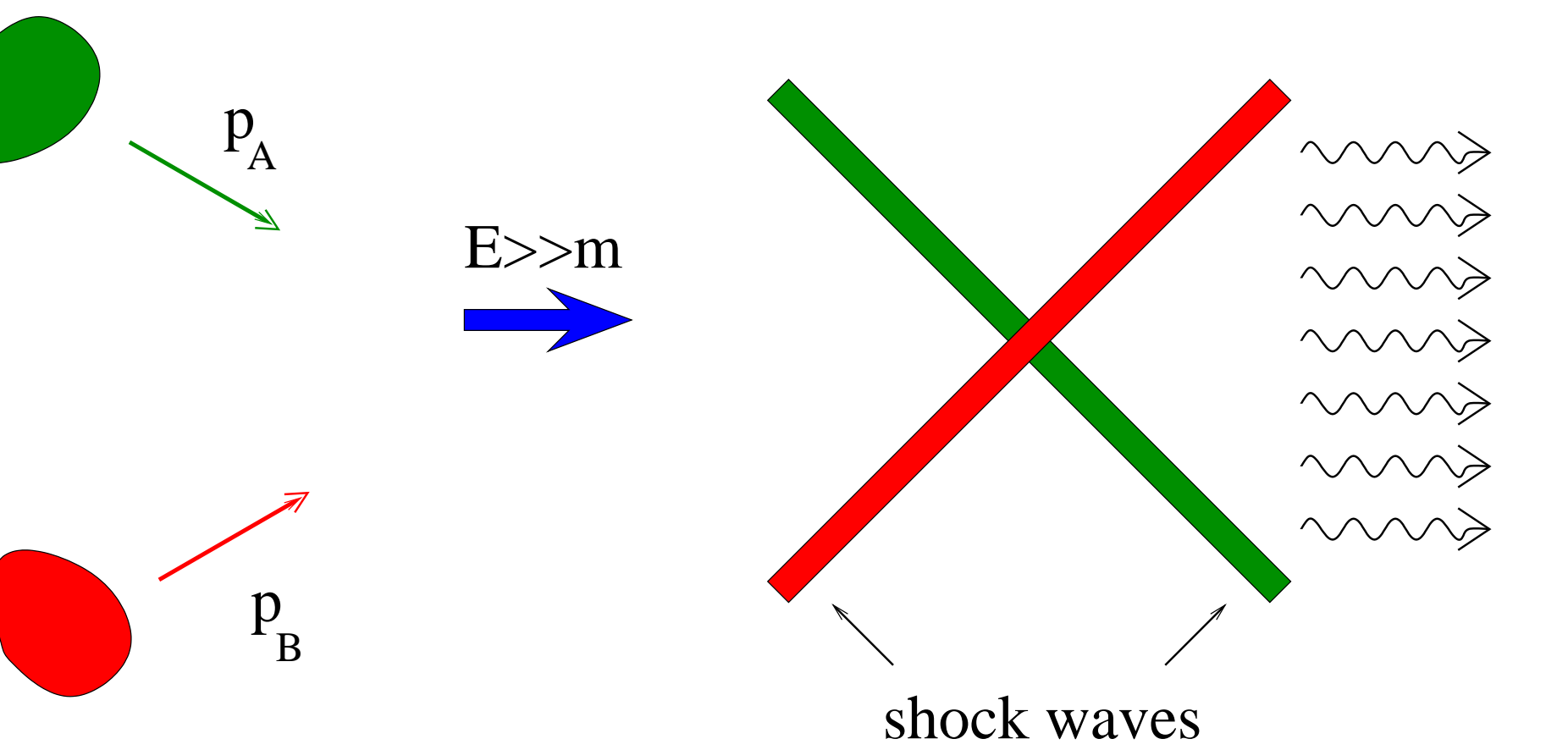

- Big Q: Produced particles/fields $\Leftrightarrow \mathbf{S}_{\mathrm{eff}}$ ? 


\section{High-energy scattering as a collision of shock waves}

A typical hadron-hadron collision viewed from the c.m. frame has the form of scattering of two shock waves.
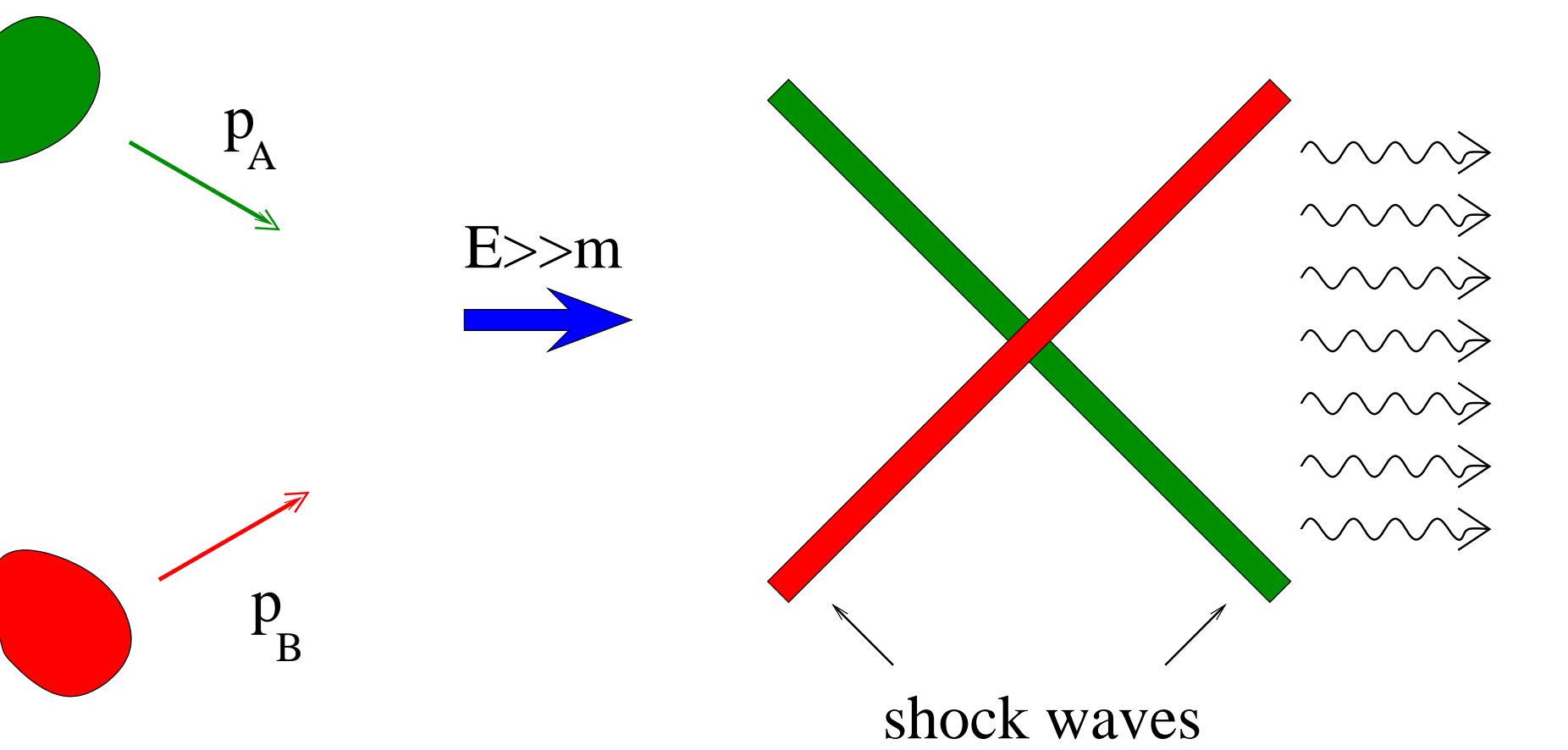

- Q \# 0: What is a scattering of two QCD shock waves?

- Big Q: Produced particles/fields $\Leftrightarrow \mathbf{S}_{\mathrm{eff}}$ ? 


\section{Rapidity factorization}

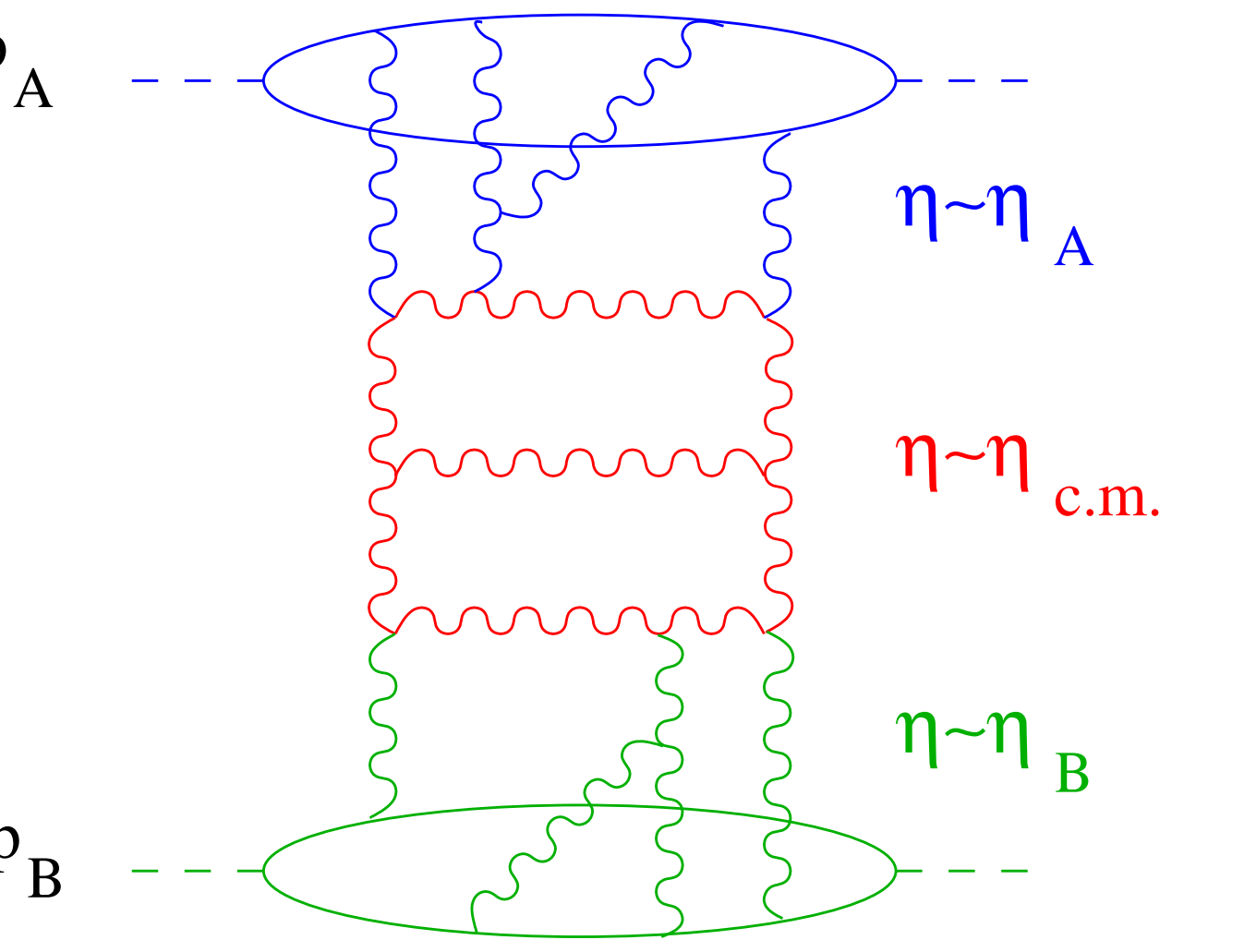

At first, we integrate over "red" gluons moving with rapidities in the central region $\eta \sim \eta_{\text {c.m. }}$.

They interact with the "external" fileds (to be integrated over later) with rapidities $\eta \sim \eta_{A}$ and $\eta \sim \eta_{B}$ 


\section{Rapidity factorization}

Consider the propagation of the red gluon in the background of blue gluons with greater rapidity 


\section{Fast-moving hadron $\Rightarrow$ QCD shock wave}
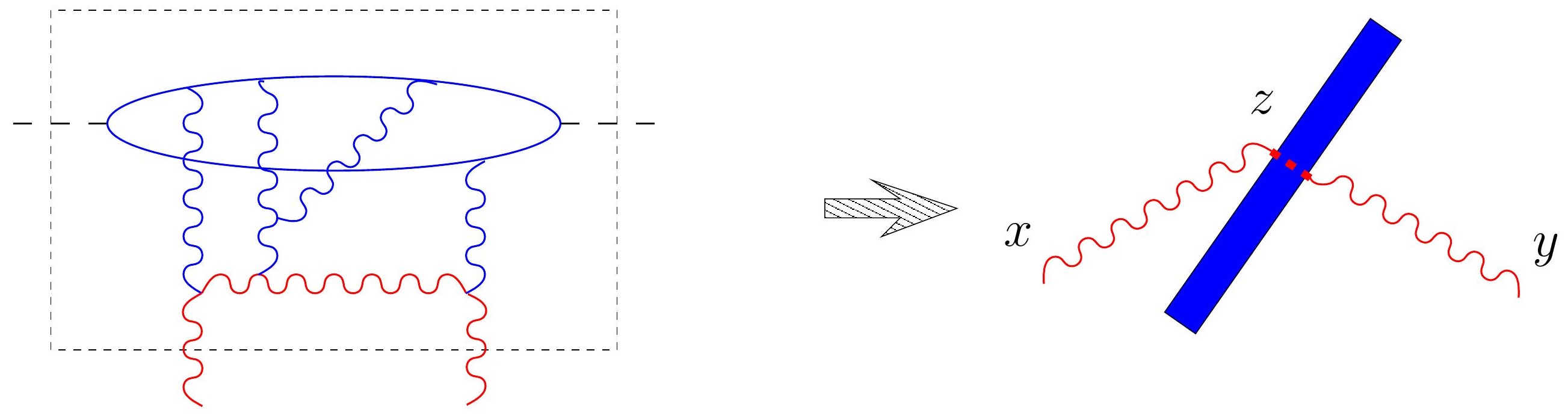

Fast-moving (blue) fileds shrink into a pancake $A_{+} \sim \delta\left(x_{-}\right)$ 


\section{Fast-moving hadron $\Rightarrow$ QCD shock wave}
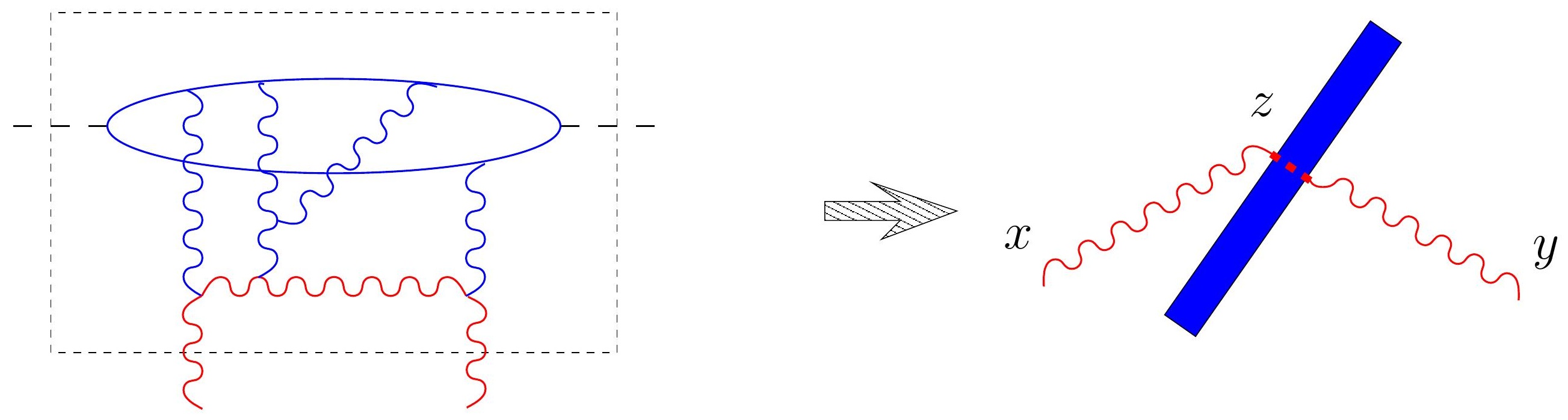

Fast-moving (blue) fileds shrink into a pancake $A_{+} \sim \delta\left(x_{-}\right)$

Interaction with the shock wave is instantaneous

$\Rightarrow$ no time to deviate in transverse plane 


\section{Fast-moving hadron $\Rightarrow$ QCD shock wave}
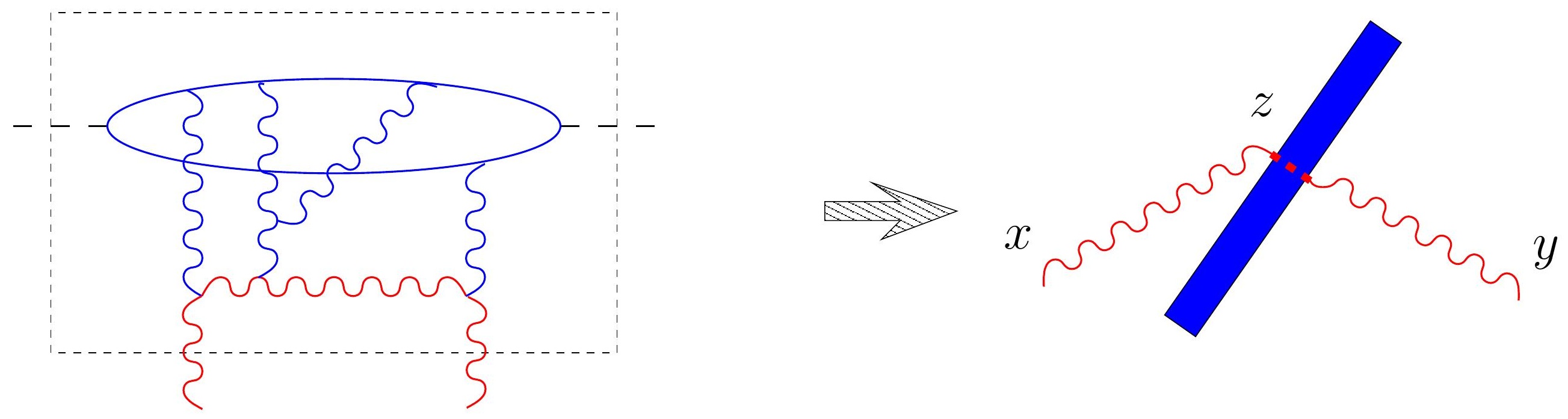

Fast-moving (blue) fileds shrink into a pancake $A_{+} \sim \delta\left(x_{-}\right)$

Interaction with the shock wave is instantaneous

$\Rightarrow$ no time to deviate in transverse plane

$\Rightarrow$ the interaction is described by the Wilson line

$$
V_{z}=\left[\infty p_{2}+z_{\perp},-\infty p_{2}+z_{\perp}\right], \quad[x, y] \equiv P e^{i g \int_{0}^{1} d u(x-y)^{\mu} A_{\mu}(u x+(1-u) y)}
$$




\section{Covariant vs axial gauge}

Covariant gauges: the shock wave is a pancake: $A_{+} \sim \delta\left(x_{-}\right), A_{-}=A_{i}=0$.
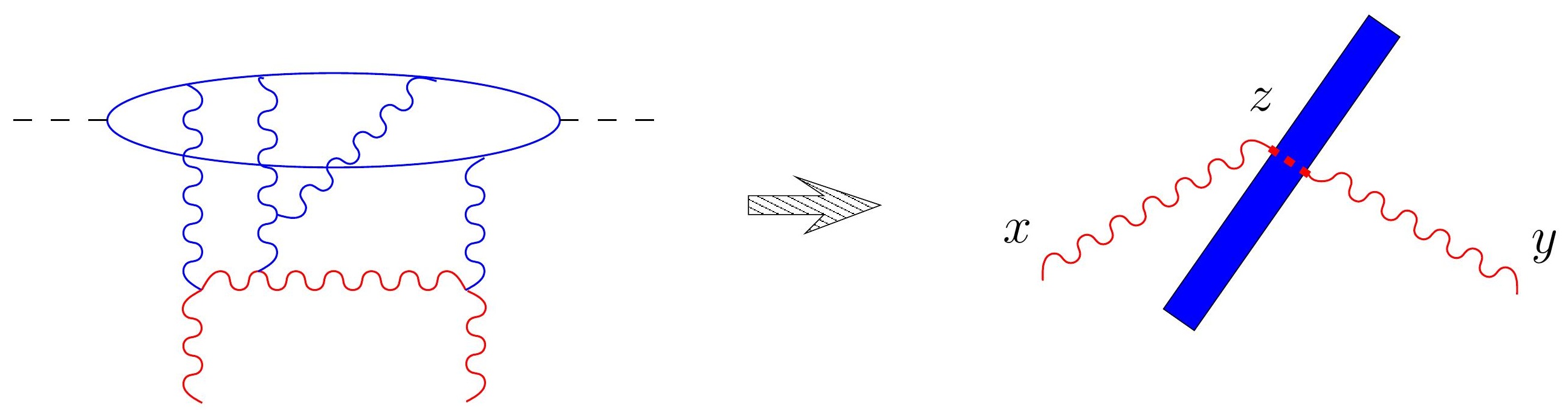


\section{Covariant vs axial gauge}

Covariant gauges: the shock wave is a pancake: $A_{+} \sim \delta\left(x_{-}\right), A_{-}=A_{i}=0$. Axial (temporal) gauges: the shock wave is a piece-wise pure gauge

$$
A^{i}=\mathcal{V}_{1}^{i}\left(z_{\perp}\right) \theta\left(z_{+}\right)+\mathcal{V}_{2}^{i}\left(z_{\perp}\right) \theta\left(-z_{+}\right), \quad A_{+}=A_{-}=0, \quad \mathcal{V}_{i} \equiv V^{\dagger} \frac{i}{g} \partial_{i} V
$$
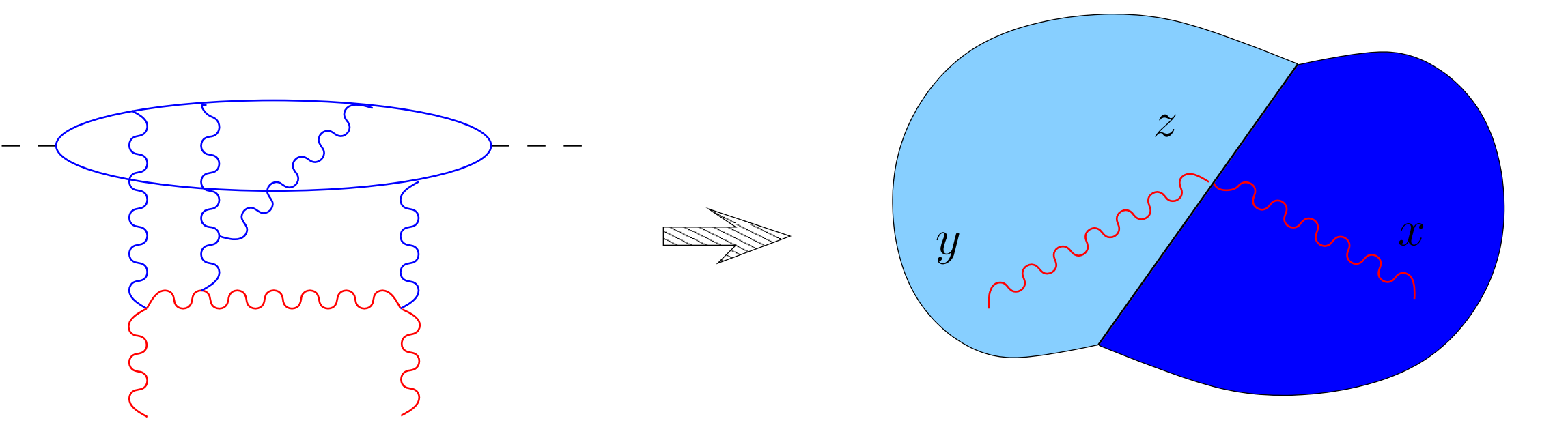


\section{Covariant vs axial gauge}

Covariant gauges: the shock wave is a pancake: $A_{+} \sim \delta\left(x_{-}\right), A_{-}=A_{i}=0$. Axial (temporal) gauges: the shock wave is a piece-wise pure gauge

$$
A^{i}=\mathcal{V}_{1}^{i}\left(z_{\perp}\right) \theta\left(z_{+}\right)+\mathcal{V}_{2}^{i}\left(z_{\perp}\right) \theta\left(-z_{+}\right), \quad A_{+}=A_{-}=0, \quad \mathcal{V}_{i} \equiv V^{\dagger} \frac{i}{g} \partial_{i} V
$$
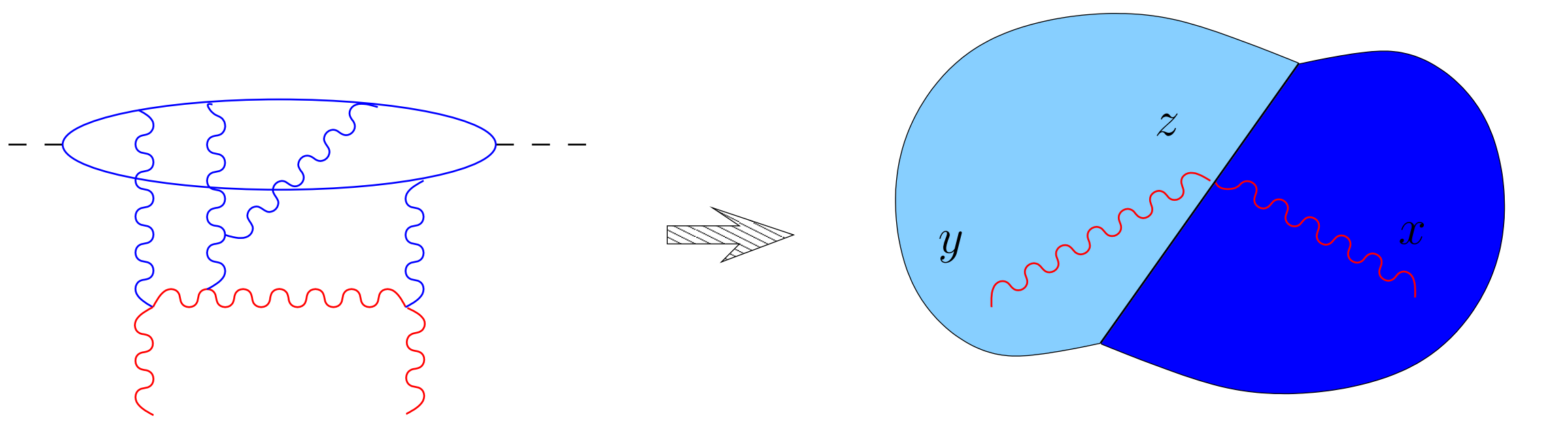

The source for such a field is

$$
\exp \left\{i \int d^{2} z_{\perp}\left\{\mathcal{V}_{1}^{i}\left(z_{\perp}\right)-\mathcal{V}_{2}^{i}\left(z_{\perp}\right)\right\}\left(0, F_{-i}, 0\right)_{z}\right\}
$$

$$
\left(0, F_{-i}, 0\right)_{z} \equiv \int d u\left[z_{\perp}, u p_{1}+z_{\perp}\right] F_{-i}\left(u p_{1}+z_{\perp}\right)\left[u p_{1}+z_{\perp}, z_{\perp}\right]
$$




\section{Second shock wave}

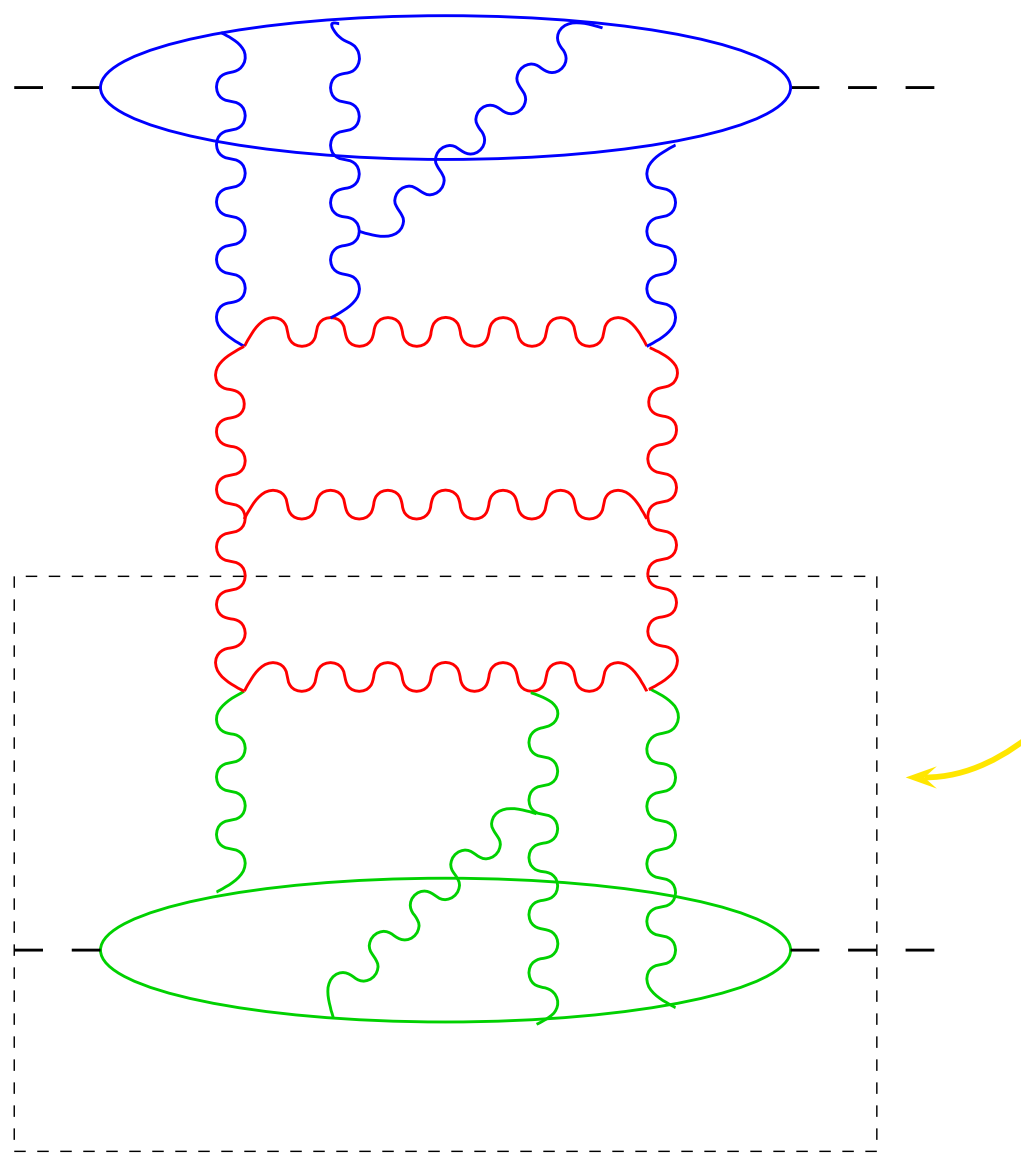

Consider now the propagation of the red gluon in the background of green gluons with greater rapidity 


\section{Second shock wave}

Covariant gauges:
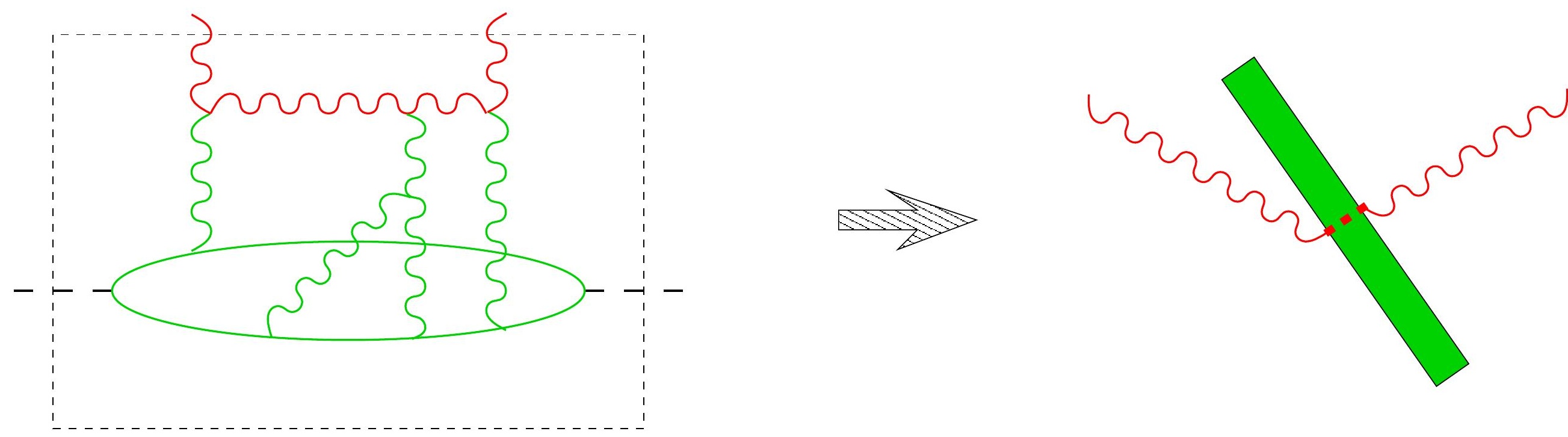


\section{Second shock wave}

Axial gauges:

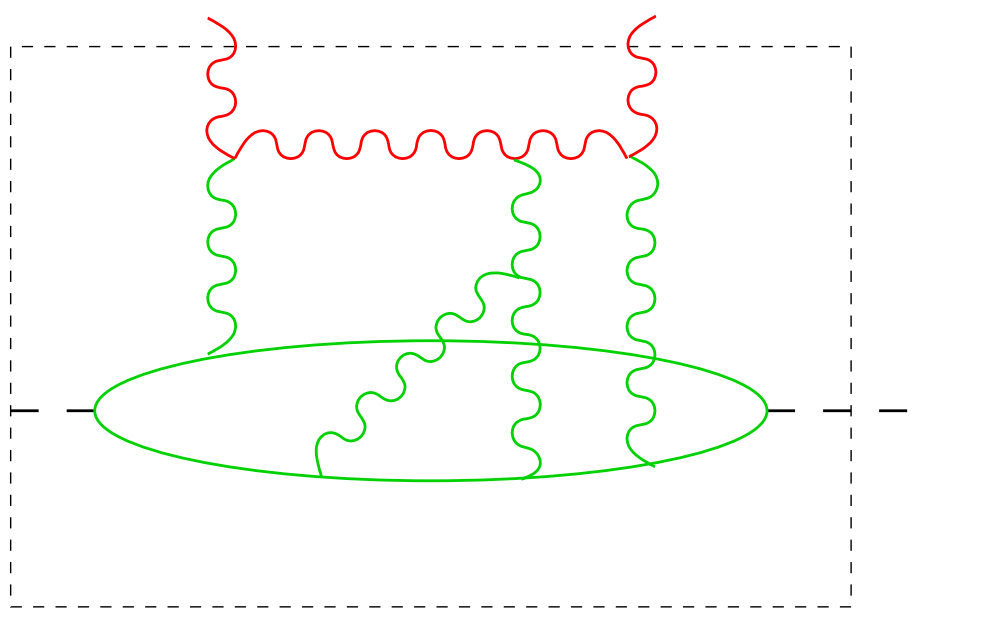

$$
\mathcal{U}_{i} \equiv U^{\dagger} \frac{i}{g} \partial_{i} U
$$

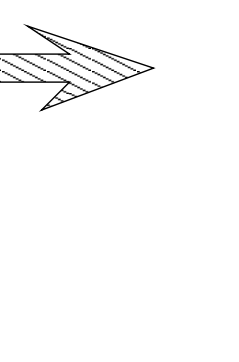

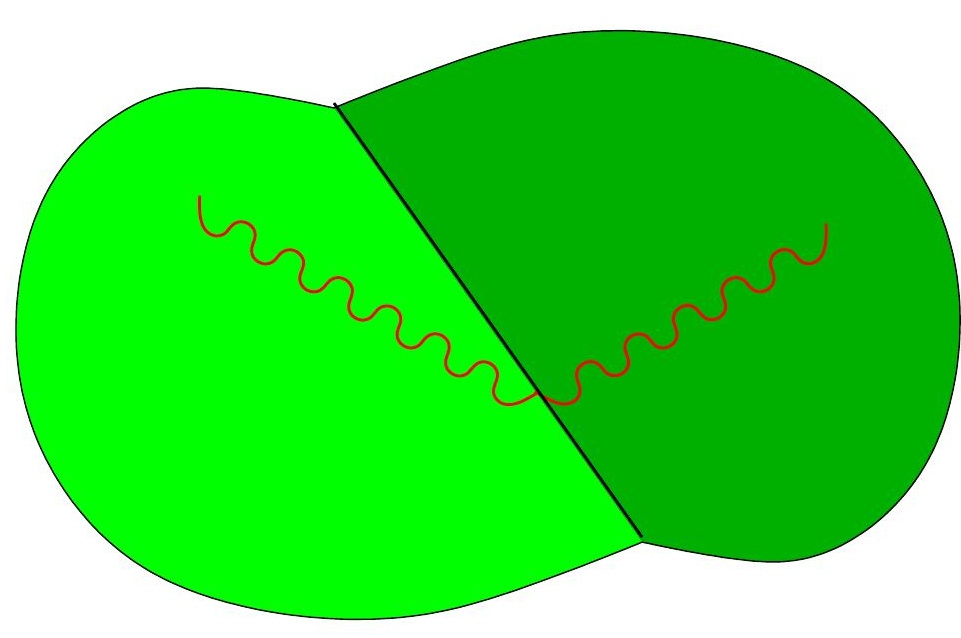

The source is

$$
\exp \left\{i \int d^{2} z_{\perp}\left(\mathcal{U}_{1}^{i}-\mathcal{U}_{2}^{i}\right)\left(z_{\perp}\right)\left(0, F_{+i}, 0\right)_{z}\right\}
$$

$\left[0, F_{+i}, 0\right] \equiv \int d u\left[z_{\perp}, u p_{2}+z_{\perp}\right] F_{+i}\left(u p_{2}+z_{\perp}\right)\left[u p_{2}+z_{\perp}, z_{\perp}\right]$

$=\left[0, \infty p_{2}\right]_{z} i \frac{\partial}{\partial z_{i}}\left[\infty p_{2}, 0\right]_{z}+\left[0,-\infty p_{2}\right]_{z} i \frac{\partial}{\partial z_{i}}\left[-\infty p_{2}, 0\right]_{z}$ 


\section{Scattering of two shock waves}

Gluons in the central region of rapidity move in the "external" fields of two shock waves

In the axial gauges
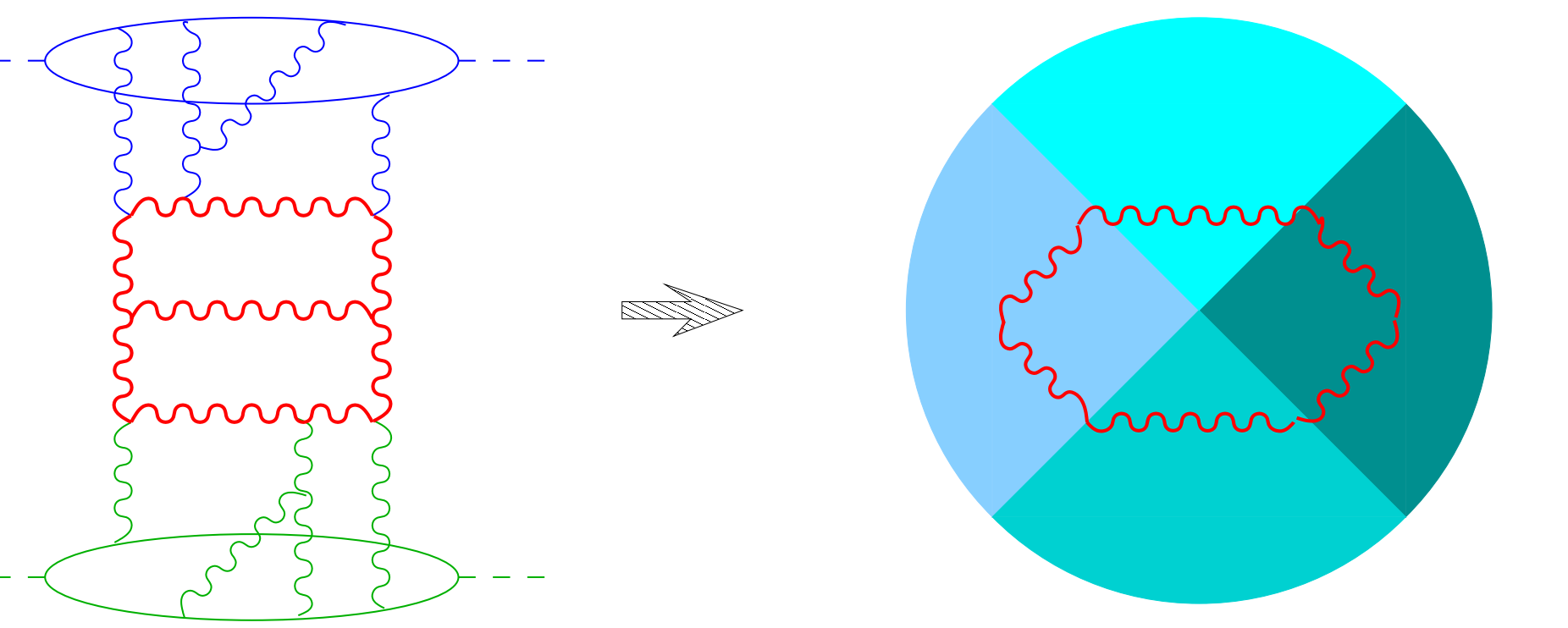


\section{Scattering of two shock waves}

Gluons in the central region of rapidity move in the "external" fields of two shock waves

In the axial gauges
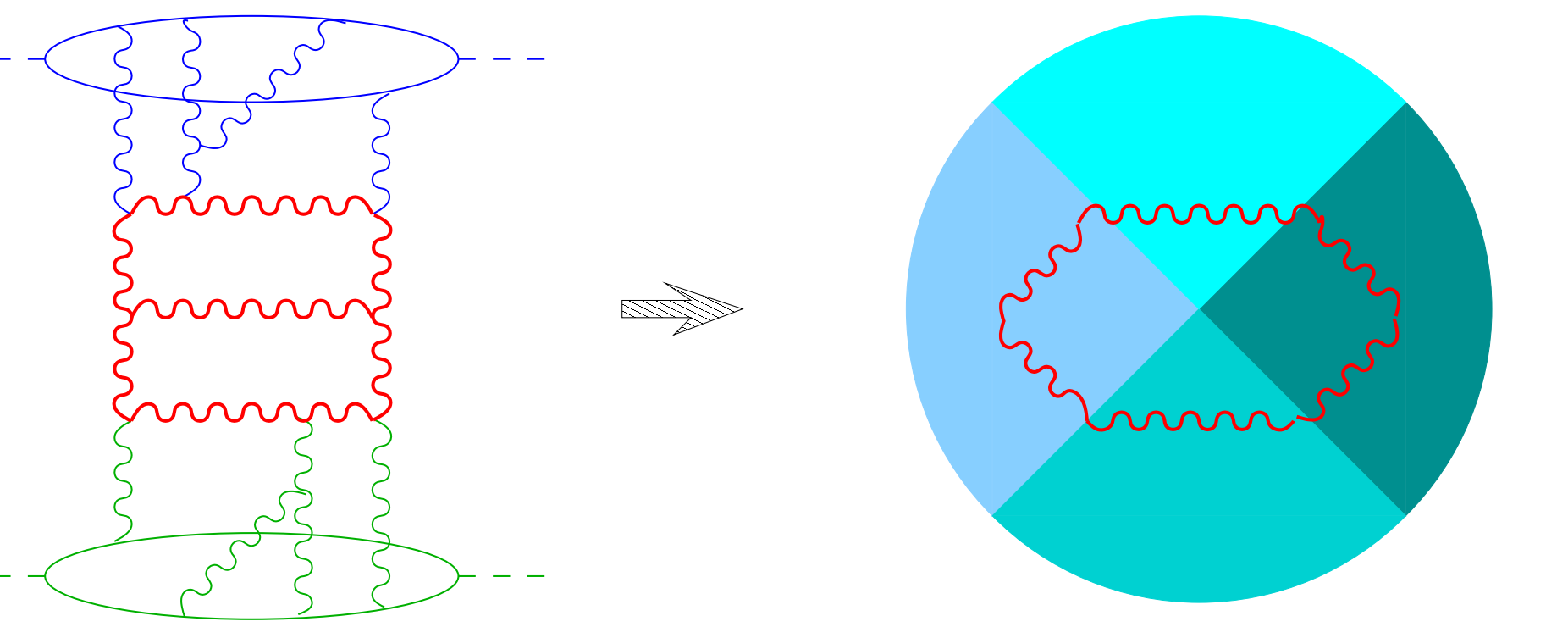

Integration over $A$ fields gives the effective action

$$
e^{i S_{\mathrm{eff}}\left(U_{i}, V_{i}, \Delta \eta\right)}=\int D A e^{i S_{\mathrm{QCD}}(A)+i \int d^{2} z_{\perp}\left\{\left(\mathcal{V}_{1}^{i}-\mathcal{V}_{2}^{i}\right)_{z}\left(0, F_{-i}, 0\right)_{z}+\left(\mathcal{U}_{1}^{i}-\mathcal{U}_{2}^{i}\right)_{z}\left[0, F_{+i}, 0\right]_{z}\right\}}
$$


Rapidity $\Leftrightarrow$ slope of the Wilson line

$$
\Delta \eta=\eta_{1}-\eta_{2}
$$
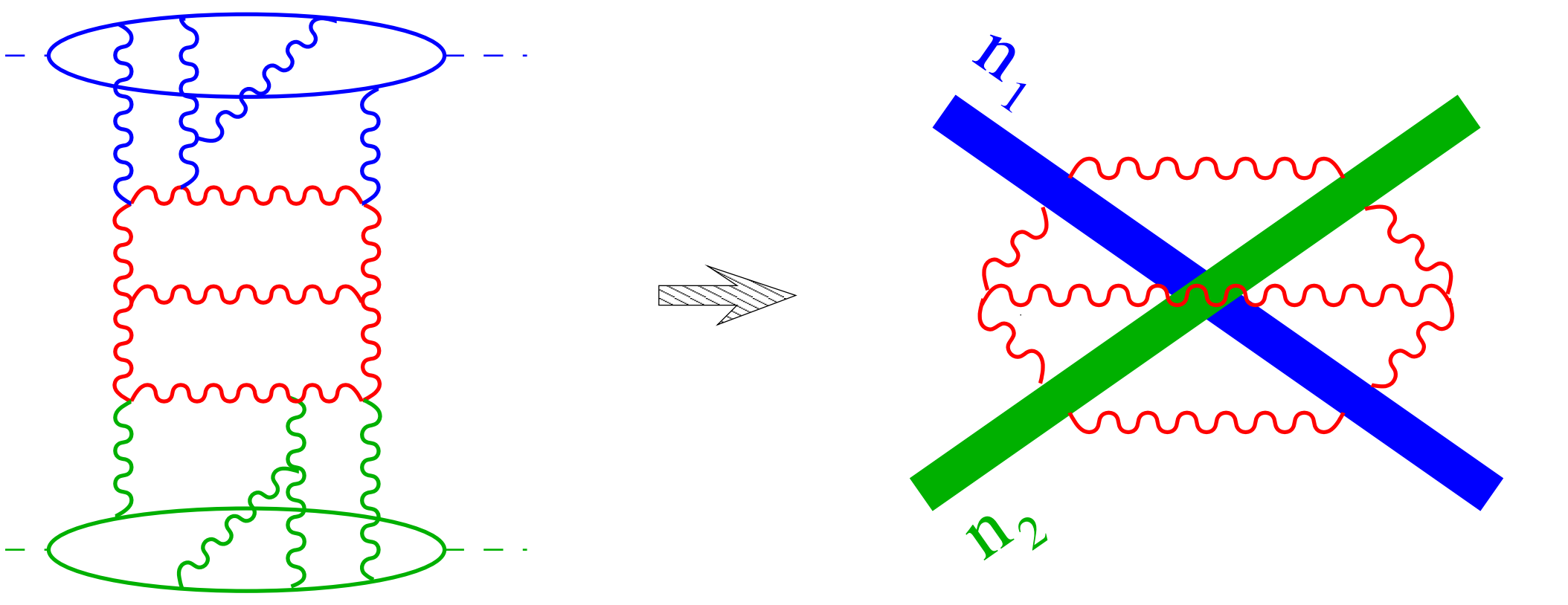

$$
e^{i S_{\mathrm{eff}}\left(U_{i}, V_{i}, \Delta \eta\right)}=\int D A e^{i S_{\mathrm{QCD}}(A)+i \int d^{2} z_{\perp}\left\{\left(\mathcal{V}_{1}^{i}-\mathcal{V}_{2}^{i}\right)_{z}\left(0, F_{-i}, 0\right)_{z}+\left(\mathcal{U}_{1}^{i}-\mathcal{U}_{2}^{i}\right)_{z}\left[0, F_{+i}, 0\right] z\right\}}
$$

$\left(0, F_{-i}, 0\right)_{z}=\left(0, \infty n_{1}\right)_{z} i \partial_{i}\left(\infty n_{1}, 0\right)_{z}+\left(0,-\infty n_{1}\right)_{z} i \partial_{i}\left(-\infty n_{1}, 0\right)_{z}$

$\left[0, F_{-i}, 0\right]_{z}=\left[0, \infty n_{2}\right]_{z} i \partial_{i}\left[\infty n_{2}, 0\right]_{z}+\left[0,-\infty n_{2}\right]_{z} i \partial_{i}\left[-\infty n_{2}, 0\right]_{z}$

$S_{\text {eff }}$ gives the small- $x$ evolution of the Wilson-line operators 


\section{Classical YM equation}

BASIC IDEA: $\alpha_{s}=\alpha_{s}\left(Q_{s}\right) \ll 1 \Rightarrow$ SEMICLASSICS IS RELEVANT

McLerran \& Venugopalan 


\section{Classical YM eque}

\section{BASIC IDEA: $\alpha_{s}=\alpha_{s}\left(Q_{s}\right) \ll 1 \Rightarrow$ SEMICLASSICS IS RELEVANT}

McLerran \& Venugopalan

$$
D^{\nu} F_{\nu \mu}=\frac{\partial}{\partial A_{\mu}}(\text { sources })
$$




\section{Classical YM equation}

BASIC IDEA: $\alpha_{s}=\alpha_{s}\left(Q_{s}\right) \ll 1 \Rightarrow$ SEMICLASSICS IS RELEVANT

McLerran \& Venugopalan

$$
D^{\nu} F_{\nu \mu}=\frac{\partial}{\partial A_{\mu}} \text { (sources) }
$$

Two methods of the solution on the market:

- Numerical simulations.

Venugopalan \& Krasnitz

- Perturbative expansion in strength of one of the shock waves

McLerran et al, Kovchegov \& Mueller 


\section{Classical YM equation}

BASIC IDEA: $\alpha_{s}=\alpha_{s}\left(Q_{s}\right) \ll 1 \Rightarrow$ SEMICLASSICS IS RELEVANT

McLerran \& Venugopalan

$$
D^{\nu} F_{\nu \mu}=\frac{\partial}{\partial A_{\mu}} \text { (sources) }
$$

Two methods of the solution on the market:

- Numerical simulations.

Venugopalan \& Krasnitz

- Perturbative expansion in strength of one of the shock waves McLerran et al, Kovchegov \& Mueller

$\Leftrightarrow$ expansion in powers of commutators $[U, V]$ (calculated up to $[U, V]^{2}$ ) 


\section{The expansion in commutators}

If $[U, V]=0$

$$
\bar{A}_{+}=\bar{A}_{-}=0, \quad \bar{A}^{i}=\mathcal{U}_{1}^{i} \theta\left(x_{+}\right)+\mathcal{U}_{2}^{i} \theta\left(-x_{+}\right)+\mathcal{V}_{1}^{i} \theta\left(x_{-}\right)+\mathcal{V}_{2}^{i} \theta\left(-x_{-}\right)
$$

$=$ piece-wise pure gauge .

QED-like: no interaction $\Rightarrow$ no particle production 


\section{The expansion in commutators}

If $[U, V]=0$

$$
\bar{A}_{+}=\bar{A}_{-}=0, \quad \bar{A}^{i}=\mathcal{U}_{1}^{i} \theta\left(x_{+}\right)+\mathcal{U}_{2}^{i} \theta\left(-x_{+}\right)+\mathcal{V}_{1}^{i} \theta\left(x_{-}\right)+\mathcal{V}_{2}^{i} \theta\left(-x_{-}\right)
$$

$=$ piece-wise pure gauge .

QED-like: no interaction $\Rightarrow$ no particle production

If $[U, V] \neq 0$ one can take this ansatz

$\bar{A}_{+}^{(0)}=\bar{A}_{-}^{(0)}=0, \quad \bar{A}^{i(0)}=\mathcal{U}_{1}^{i} \theta\left(x_{+}\right)+\mathcal{U}_{2}^{i} \theta\left(-x_{+}\right)+\mathcal{V}_{1}^{i} \theta\left(x_{-}\right)+\mathcal{V}_{2}^{i} \theta\left(-x_{-}\right)$

as a trial configuration for the classical solution and improve it order by order in $[U, V]$ by calculating Feynman diagrams in the background of the trial configuration. 


\section{The expansion in commutators}

Solve the YM eqn for $Q_{\mu}(x)$ by iterations $\Leftrightarrow$ calculate Feynman diagrams in the external field $\bar{A}^{(0)}$

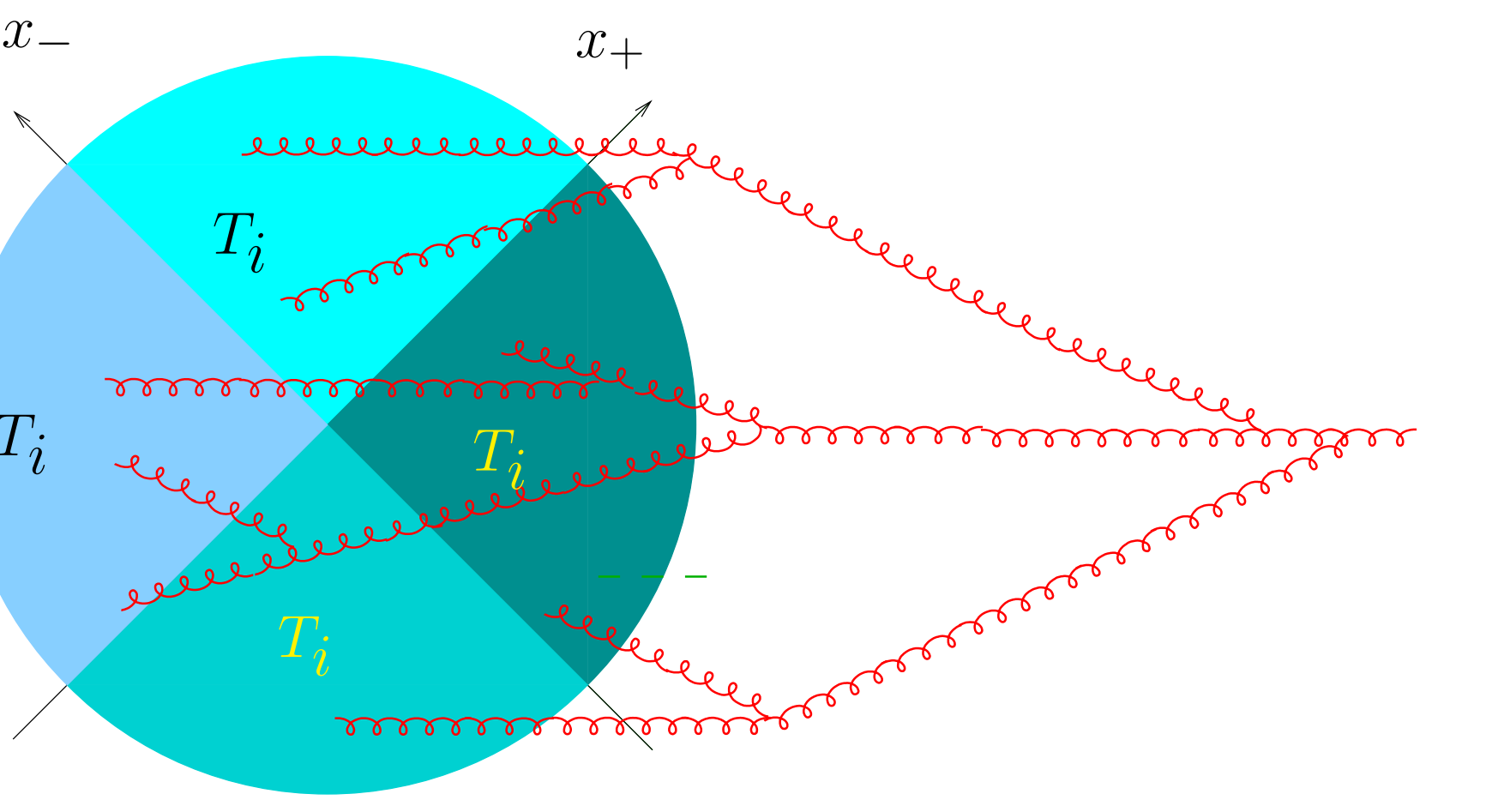

$T$ - linear term

$T_{\mu}=D^{\nu} \bar{F}_{\nu \mu}^{(0)}-\frac{\partial}{\partial A_{\mu}}$ (sources) 


\section{Zero-order approximation: a piece-wise pure gauge field}

$$
\begin{aligned}
\bar{A}_{+} & =\bar{A}_{-}=0 \\
\bar{A}^{i} & =\mathcal{W}_{F}^{i} \theta\left(x_{+}\right) \theta\left(x_{-}\right)+\mathcal{W}_{L}^{i} \theta\left(-x_{+}\right) \theta\left(x_{-}\right) \\
& +\mathcal{W}_{R}^{i} \theta\left(x_{+}\right) \theta\left(-x_{-}\right)+\mathcal{W}_{B}^{i} \theta\left(-x_{+}\right) \theta\left(-x_{-}\right) \\
\mathcal{W}_{F}^{i}\left(x_{\perp}\right) & =\mathcal{U}_{1}^{i}+\mathcal{V}_{1}^{i}+E_{F}^{i}=\text { pure gauge } \\
\mathcal{W}_{L}^{i}\left(x_{\perp}\right) & =\mathcal{U}_{2}^{i}+\mathcal{V}_{1}^{i}+E_{L}^{i}=\ldots \\
\mathcal{W}_{R}^{i}\left(x_{\perp}\right) & =\mathcal{U}_{1}^{i}+\mathcal{V}_{2}^{i}+E_{R}^{i}=\ldots \\
\mathcal{W}_{B}^{i}\left(x_{\perp}\right) & =\mathcal{U}_{2}^{i}+\mathcal{V}_{2}^{i}+E_{B}^{i}=\ldots
\end{aligned}
$$

In the first order

$$
\begin{gathered}
E_{i}^{a}\left(x_{\perp}\right)=i g \int d^{2} z\left(U_{x} U_{z}^{\dagger}+V_{x} V_{z}^{\dagger}-1\right)^{a b} \frac{(x-z)_{\perp}^{k}}{2 \pi^{2}(x-z)_{\perp}^{2}}\left(\left[\mathcal{U}_{i}, \mathcal{V}_{k}\right]_{z}-i \leftrightarrow k\right)^{b} \\
\text { bF gauge } D^{\mu} Q_{\mu}=0 \rightarrow\left(i \partial_{i}+g\left[\mathcal{U}_{i}+\mathcal{V}_{i},\right) E^{i}=0\right.
\end{gathered}
$$




\section{$Q_{\mu}$ in the first order}

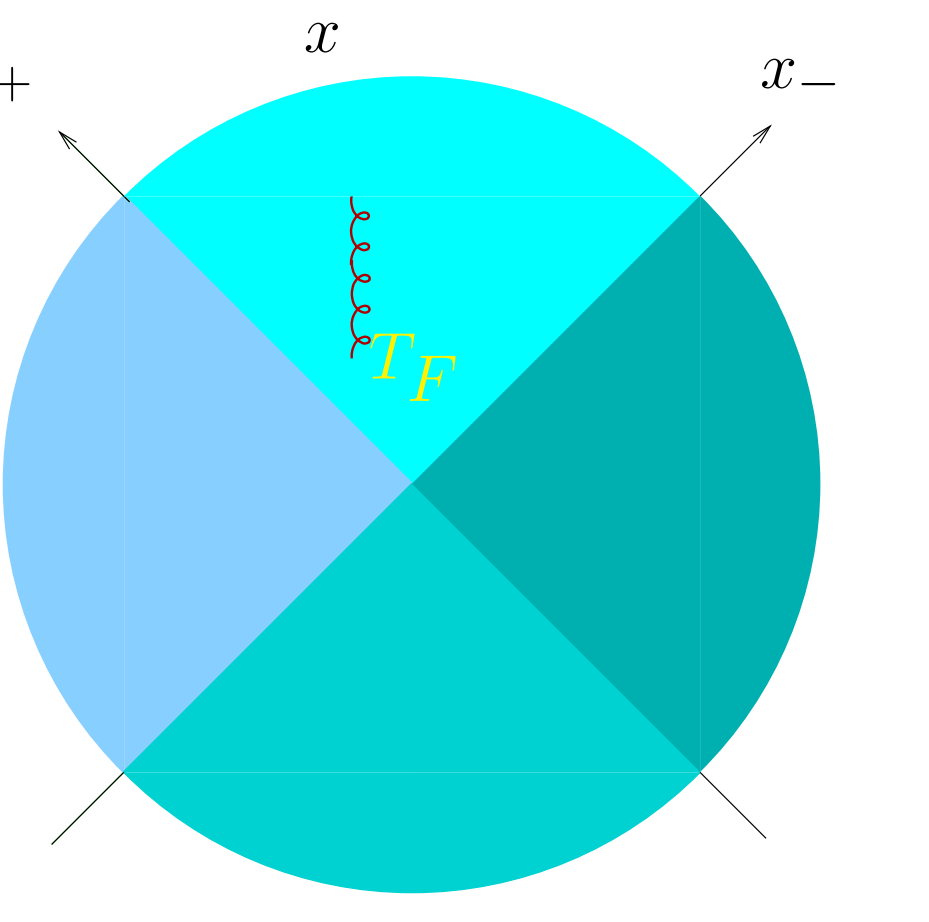




\section{$Q_{\mu}$ in the first order}

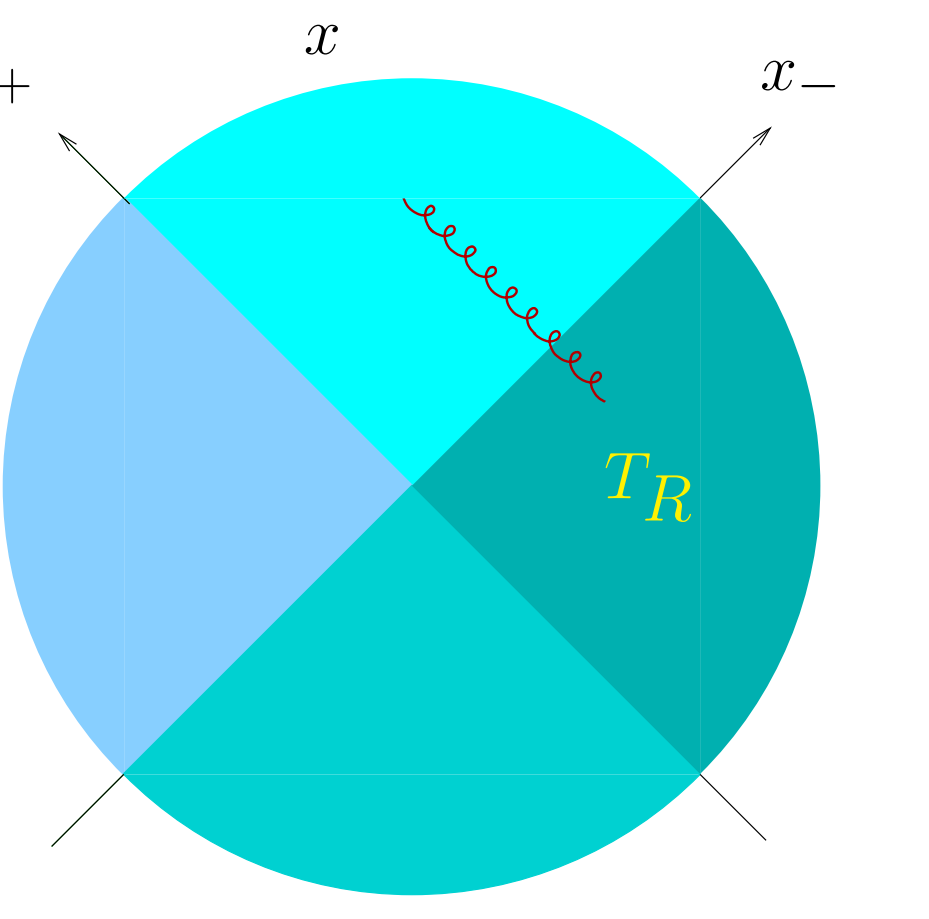




\section{$Q_{\mu}$ in the first order}

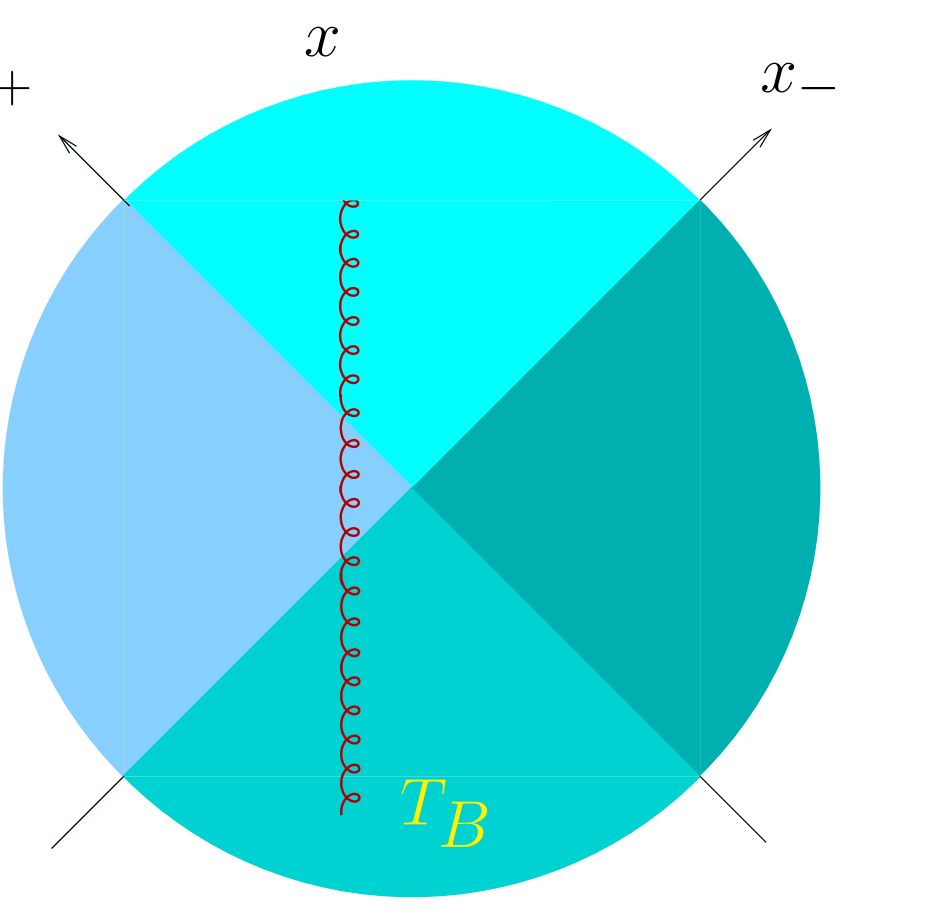




\section{$Q_{\mu}$ in the first order}

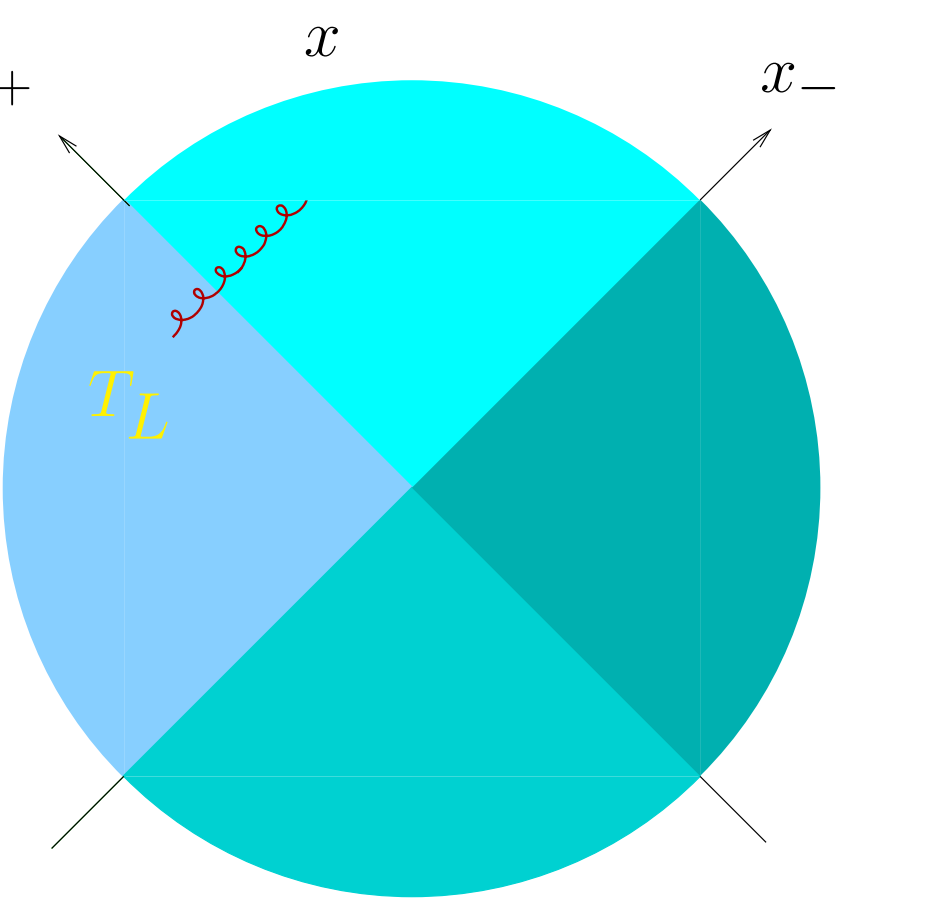




\section{$Q_{\mu}$ in the first order}

Lipatov vertex (effective vertex of gluon emission):

$$
\begin{aligned}
& L_{\mu}^{(1)}(k)=\left.k^{2} Q_{(1) \mu}^{\mathcal{W}_{F}}(k)\right|_{k^{2}=0}= \\
& 2 E_{\perp}^{\mu}+2 \frac{p_{1}^{\mu}}{k_{-}}\left[\mathcal{V}_{1 i}-\mathcal{V}_{2 i}, E_{R}^{i}-E_{2}^{i}\right]+2 \frac{p_{2}^{\mu}}{k_{+}}\left[\mathcal{U}_{1 i}-\mathcal{U}_{2 i}, E_{L}^{i}-E_{2}^{i}\right]
\end{aligned}
$$

Effective action = product of two Lipatov vertices. In the $[U, V]^{2}$ order

$$
L_{\mu}^{a} L^{a \mu}=4 E_{a}^{i} E^{a i}
$$


The trial configuration:

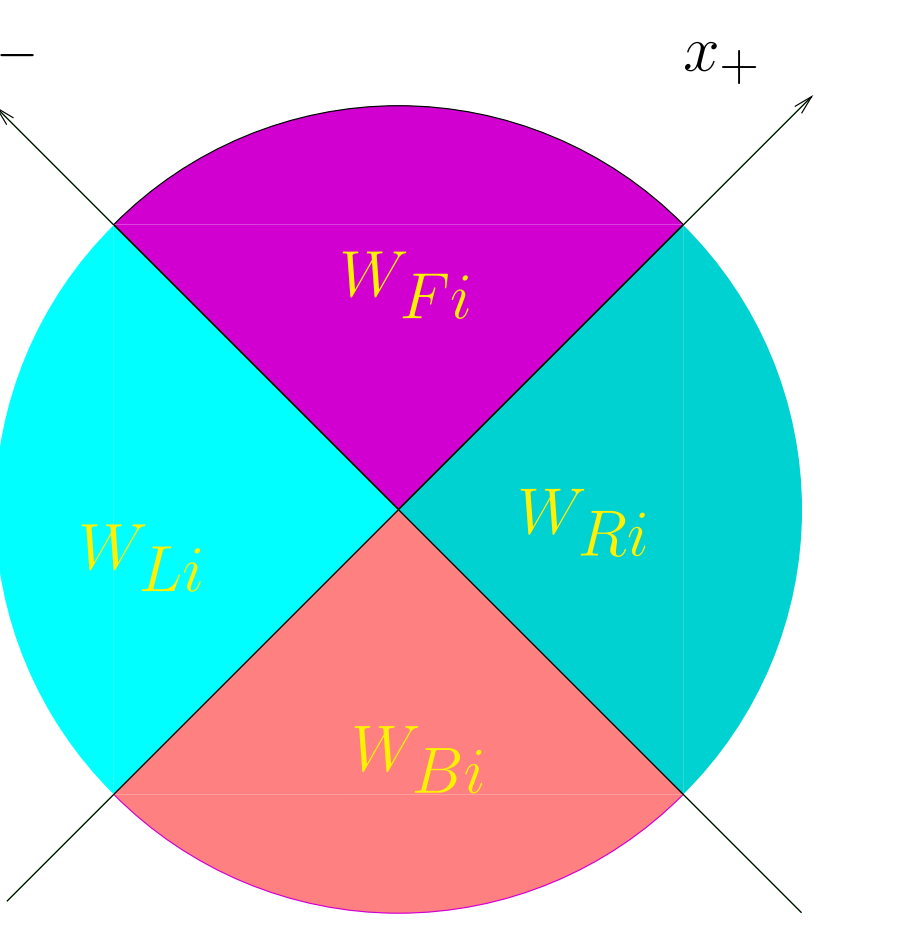

$$
\begin{aligned}
& A_{-}=A_{+}=0 \text { and } \\
& A_{i}=\theta\left(x_{+}\right) \theta\left(x_{-}\right) \mathcal{W}_{F i}+\theta\left(x_{+}\right) \theta\left(-x_{+}\right) \mathcal{W}_{R} \\
& +\theta\left(-x_{+}\right) \theta\left(x_{-}\right) \mathcal{W}_{L i}+\theta\left(-x_{+}\right) \theta\left(-x_{-}\right) \mathcal{W}_{B}
\end{aligned}
$$

In each of the four quadrants of the space the field is a pure gauge

$$
\begin{aligned}
& \mathcal{W}_{F i}=\mathcal{U}_{1 i}+\mathcal{V}_{1 i}+E_{F i} \\
& \mathcal{W}_{L i}=\mathcal{U}_{2 i}+\mathcal{V}_{1 i}+E_{L i} \\
& \mathcal{W}_{R i}=\mathcal{U}_{1 i}+\mathcal{V}_{2 i}+E_{R i} \\
& \mathcal{W}_{B i}=\mathcal{U}_{2 i}+\mathcal{V}_{2 i}+E_{F i}
\end{aligned}
$$


The trial configuration:

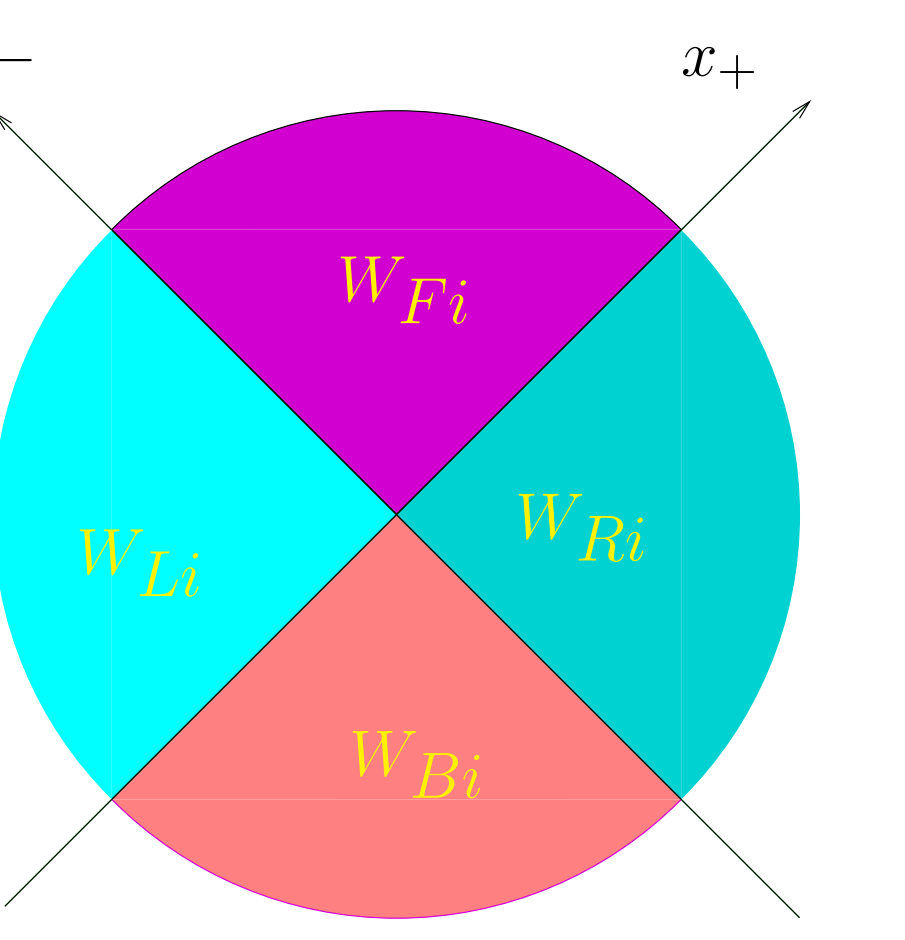

$$
\begin{aligned}
& A_{-}=A_{+}=0 \text { and } \\
& A_{i}=\theta\left(x_{+}\right) \theta\left(x_{-}\right) \mathcal{W}_{F i}+\theta\left(x_{+}\right) \theta\left(-x_{+}\right) \mathcal{W}_{R} \\
& +\theta\left(-x_{+}\right) \theta\left(x_{-}\right) \mathcal{W}_{L i}+\theta\left(-x_{+}\right) \theta\left(-x_{-}\right) \mathcal{W}_{B}
\end{aligned}
$$

In each of the four quadrants of the space the field is a pure gauge

$$
\begin{aligned}
& \mathcal{W}_{F i}=\mathcal{U}_{1 i}+\mathcal{V}_{1 i}+E_{F i} \\
& \mathcal{W}_{L i}=\mathcal{U}_{2 i}+\mathcal{V}_{1 i}+E_{L i} \\
& \mathcal{W}_{R i}=\mathcal{U}_{1 i}+\mathcal{V}_{2 i}+E_{R i} \\
& \mathcal{W}_{B i}=\mathcal{U}_{2 i}+\mathcal{V}_{2 i}+E_{F i}
\end{aligned}
$$

$T_{i}=2 \delta\left(x_{+}\right) \delta\left(x_{-}\right) E_{i} \Rightarrow$

$$
S_{\mathrm{eff}}=\int d z d z^{\prime} T_{i}^{a}(z) G^{a b}\left(z, z^{\prime}\right) T^{b i}\left(z^{\prime}\right) \simeq \alpha_{s} \Delta \eta \int d^{2} z_{\perp} E_{i}^{a}\left(z_{\perp}\right) E^{a i}\left(z_{\perp}\right)
$$




\section{Gauge-invariant form of the effective action}

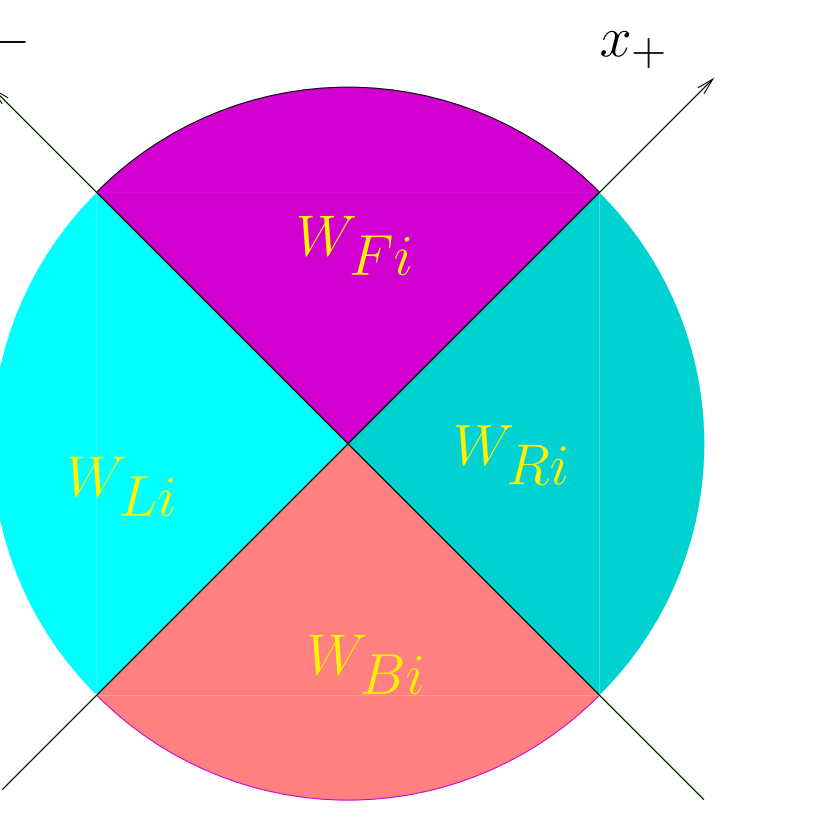

$$
\begin{aligned}
& S_{\text {eff }}\left(V_{1}, V_{2}, U_{1}, U_{2} ; \Delta \eta\right)= \\
& \begin{aligned}
\left(\mathcal{V}_{1}-\mathcal{V}_{2}\right)^{a i}\left(\mathcal{U}_{1}-\mathcal{U}_{2}\right)_{i}^{a}+\frac{\alpha_{s} \Delta \eta}{4} L_{i}^{a} L^{a i} \\
L_{i}^{a}=2\left(\mathcal{W}_{F}-\mathcal{W}_{L}-\mathcal{W}_{R}+\mathcal{W}_{B}\right)_{i}^{a} \\
\quad=2\left(E_{F}-E_{L}-E_{R}+E_{B}\right)_{i}^{a}
\end{aligned}
\end{aligned}
$$




\section{Gauge-invariant form of the effective action}

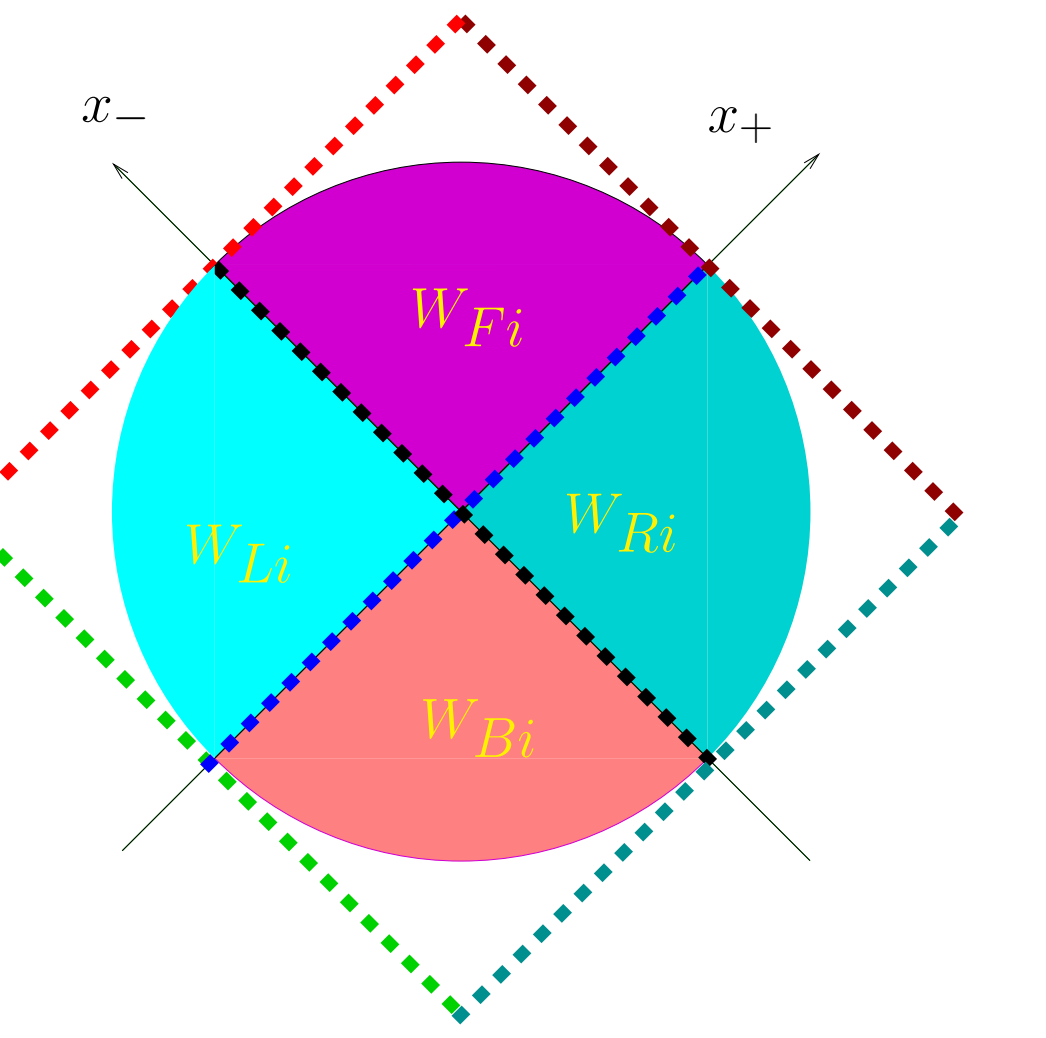

$$
\begin{aligned}
& S_{\mathrm{eff}}\left(V_{1}, V_{2}, U_{1}, U_{2} ; \Delta \eta\right)= \\
& \begin{aligned}
\left(\mathcal{V}_{1}-\mathcal{V}_{2}\right)^{a i}\left(\mathcal{U}_{1}-\mathcal{U}_{2}\right)_{i}^{a}+\frac{\alpha_{s} \Delta \eta}{4} L_{i}^{a} L^{a i} \\
L_{i}^{a}=2\left(\mathcal{W}_{F}-\mathcal{W}_{L}-\mathcal{W}_{R}+\mathcal{W}_{B}\right)_{i}^{a} \\
\quad=2\left(E_{F}-E_{L}-E_{R}+E_{B}\right)_{i}^{a}
\end{aligned}
\end{aligned}
$$

Gauge invariant representation (HIMST):

$$
\begin{aligned}
\frac{1}{4} L^{a i} L_{i}^{a} & =\operatorname{tr}\left[\infty p_{1}, F_{-i},-\infty p_{1}\right]_{\infty p_{2}}\left[\infty p_{2}, F_{+i},-\infty p_{2}\right]_{\infty p_{1}} \\
& \times\left[\infty p_{1},-\infty p_{1}\right]_{-\infty p_{2}}\left[-\infty p_{2}, \infty p_{2}\right]_{-\infty p_{1}}+\text { cyclic perm. }
\end{aligned}
$$

$\left[\infty p_{1}, F_{-i},-\infty p_{1}\right] \equiv \int_{-\infty}^{\infty} d u\left[\infty p_{1}, u p_{1}\right] F_{-i}\left(u p_{1}\right)\left[u p_{1},-\infty p_{1}\right]$ 


\section{Gauge-invariant form of the effective action}

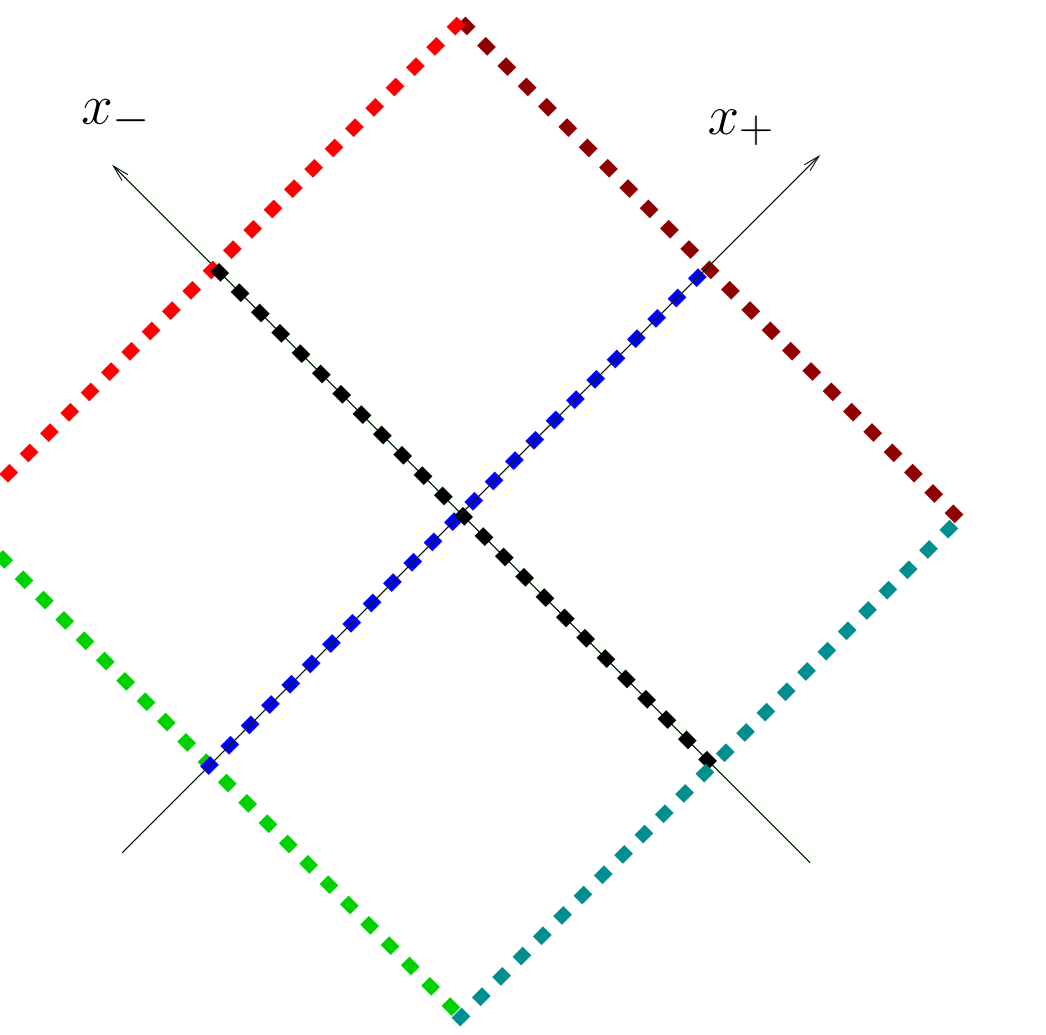

$$
\begin{aligned}
& S_{\mathrm{eff}}\left(V_{1}, V_{2}, U_{1}, U_{2} ; \Delta \eta\right)= \\
& \begin{aligned}
\left(\mathcal{V}_{1}-\mathcal{V}_{2}\right)^{a i}\left(\mathcal{U}_{1}-\mathcal{U}_{2}\right)_{i}^{a}+\frac{\alpha_{s} \Delta \eta}{4} L_{i}^{a} L^{a i} \\
L_{i}^{a}=2\left(\mathcal{W}_{F}-\mathcal{W}_{L}-\mathcal{W}_{R}+\mathcal{W}_{B}\right)_{i}^{a} \\
\quad=2\left(E_{F}-E_{L}-E_{R}+E_{B}\right)_{i}^{a}
\end{aligned}
\end{aligned}
$$

Gauge invariant representation (HIMST):

$$
\begin{aligned}
\frac{1}{4} L^{a i} L_{i}^{a} & =\operatorname{tr}\left[\infty p_{1}, F_{-i},-\infty p_{1}\right]_{\infty p_{2}}\left[\infty p_{2}, F_{+i},-\infty p_{2}\right]_{\infty p_{1}} \\
& \times\left[\infty p_{1},-\infty p_{1}\right]_{-\infty p_{2}}\left[-\infty p_{2}, \infty p_{2}\right]_{-\infty p_{1}}+\text { cyclic perm. }
\end{aligned}
$$

$\left[\infty p_{1}, F_{-i},-\infty p_{1}\right] \equiv \int_{-\infty}^{\infty} d u\left[\infty p_{1}, u p_{1}\right] F_{-i}\left(u p_{1}\right)\left[u p_{1},-\infty p_{1}\right]$ 


\section{Functional integral over the Wilson-line variables}
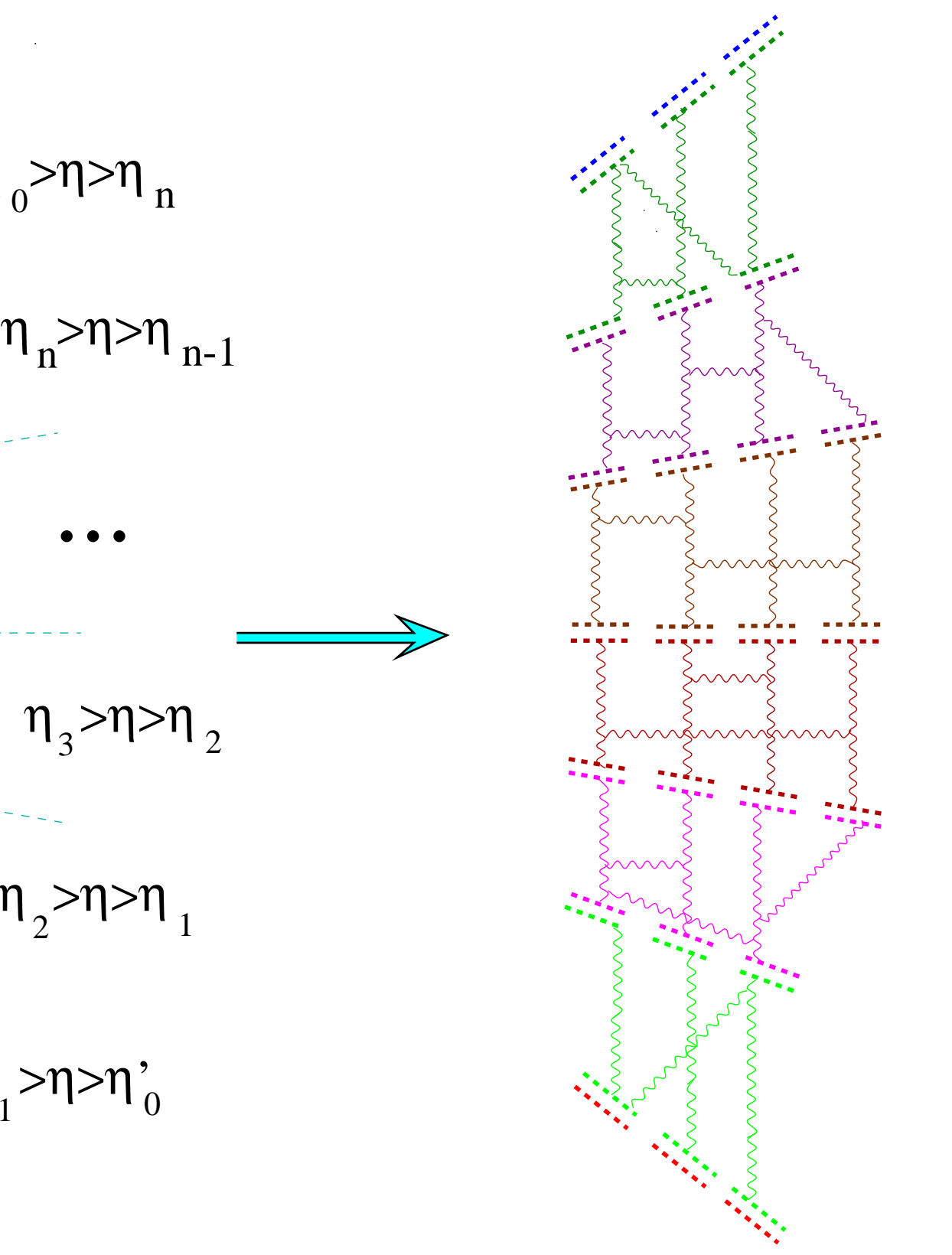


\section{Functional integral over the Wilson-line variables}

$e^{i S_{\mathrm{eff}}\left(U_{1}, U_{2}, V_{1}, V_{2} ; \eta_{1}-\eta_{2}\right)}=$

$\int_{U_{\eta_{2}}=U} D U_{1 x}^{\eta} D U_{2 x}^{\eta} D V_{1 x}^{\eta} D V_{2 x}^{\eta} e^{i \int d^{2} x_{\perp}\left[\left(\mathcal{V}_{1}-\mathcal{V}_{2}\right)_{i}^{a}\left(\mathcal{U}_{1}^{\eta_{1}}-\mathcal{U}_{2}^{\eta_{1}}\right)^{a i}+\int_{\eta_{2}}^{\eta_{1}} d \eta \mathcal{L}\left(U_{1}, U_{2}, V_{1}, V_{2}, \eta\right)\right]}$

$\left.\left.\mathcal{L}\left(U_{k}, V_{k}, \eta\right)=-\left(\mathcal{V}_{1}^{\eta}-\mathcal{V}_{2}^{\eta}\right)_{i}^{a} \frac{\partial}{\partial \eta}\left(\mathcal{U}_{1}^{\eta}-\mathcal{U}_{2}^{\eta}\right)^{a i}-i \frac{\alpha_{s}}{4} L_{i}^{a}(U, V) L^{a i}(U, V)\right]\right\}$

$L_{i}$ is local in terms of $W$ 's but unfortunately non-local in terms of $U$ and $V$.

This formula contains both "upside down" and "bottom up" small- $x$ "fan" evolutions $\Rightarrow$ pomeron loops

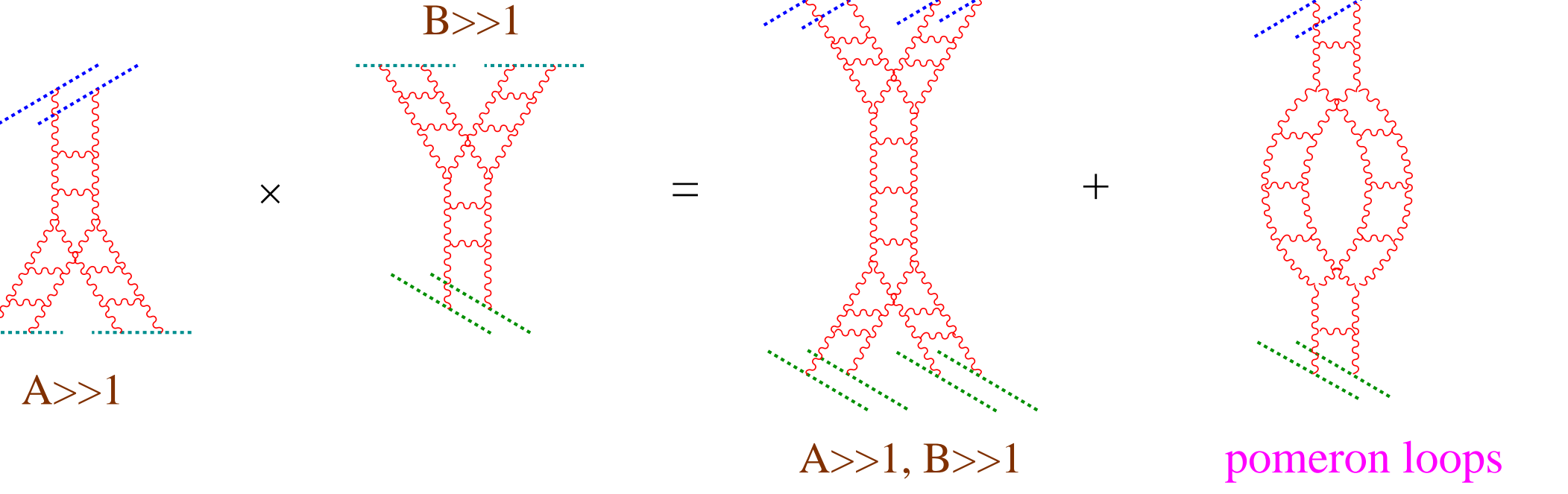




\section{Conclusions}

- High-energy hadron-hadron scattering $\Leftrightarrow$ collision of two QCD shock waves (Color Glass Condensates?)

- For two nuclei, $A$ and $B$, the expansion in commutators of Wilson lines is a symmetic expansion in both $\frac{B}{A}$ or $\frac{A}{B}$ parameters.

- $\mathcal{L}(U, V) \ni$ pomeron loops ( $\Rightarrow$ unitarity?) 


\section{Conclusions}

- High-energy hadron-hadron scattering $\Leftrightarrow$ collision of two QCD shock waves (Color Glass Condensates?)

- For two nuclei, $A$ and $B$, the expansion in commutators of Wilson lines is a symmetic expansion in both $\frac{B}{A}$ or $\frac{A}{B}$ parameters.

- $\mathcal{L}(U, V) \ni$ pomeron loops ( $\Rightarrow$ unitarity?)

Outlook

- The $[U, V]^{2}$ term in $\mathcal{L}$

- Big Q: What is the field produced by the collision (in all orders in $[U, V])$ ?

- $\Leftrightarrow$ Big Q: $S_{\text {eff }}$ in (in all orders in $[U, V]$ ) ? 


\section{Addendum}

An example of Feynman diagram not taken into account by the diamond action

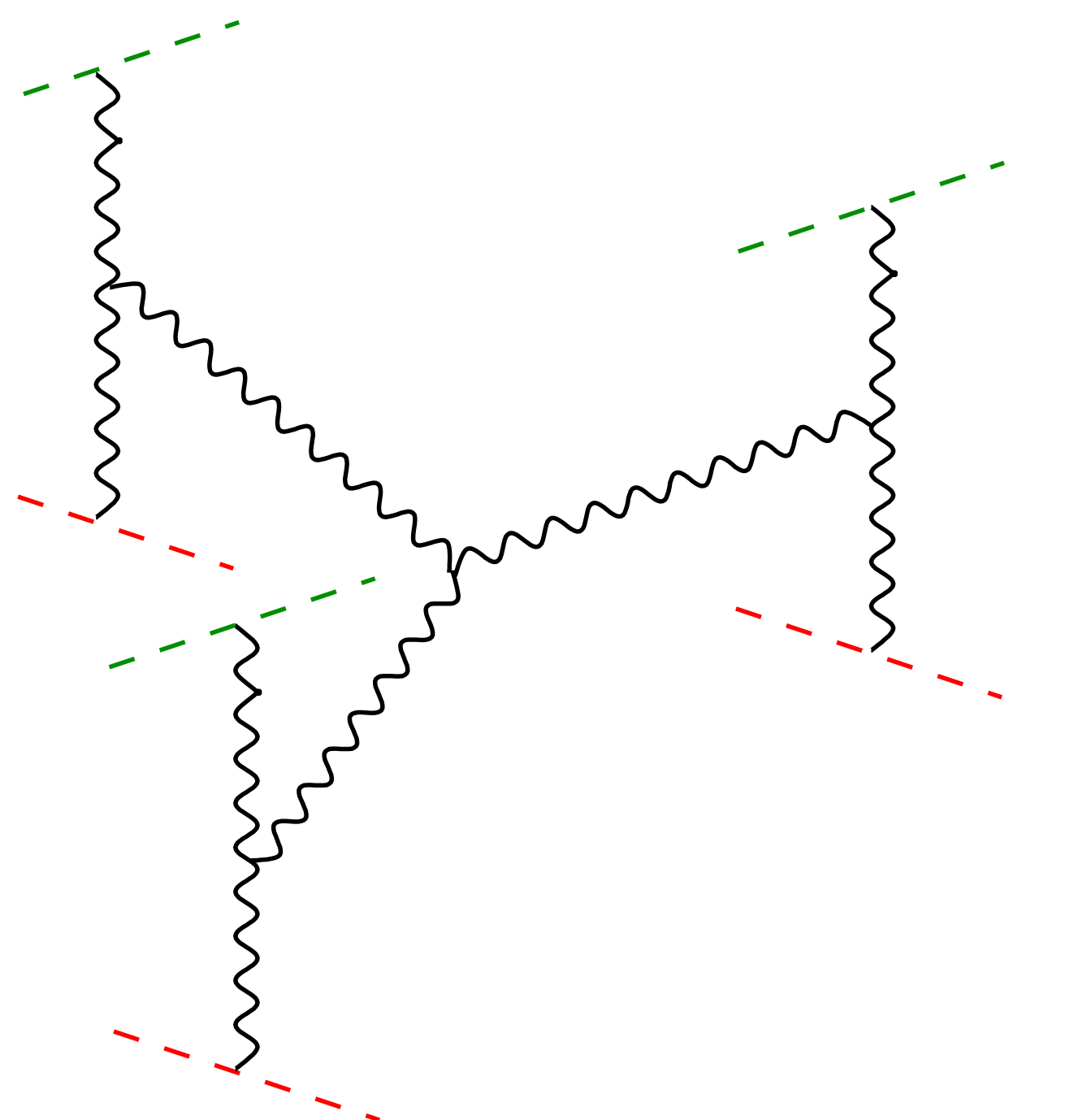

